\title{
A mathematical analysis of a system of Caputo-Fabrizio fractional differential equations for the anthrax disease model in animals
}

\author{
Shahram Rezapour ${ }^{1,2,3}$, Sina Etemad ${ }^{4}$ and Hakimeh Mohammadi ${ }^{*}$
}

\author{
"Correspondence: \\ hakimeh.mohammadi@yahoo.com \\ ${ }^{5}$ Department of Mathematics, \\ Miandoab Branch, Islamic Azad \\ University, Miandoab, Iran \\ Full list of author information is \\ available at the end of the article
}

\begin{abstract}
We study a fractional-order model for the anthrax disease between animals based on the Caputo-Fabrizio derivative. First, we derive an existence criterion of solutions for the proposed fractional $\mathcal{C F}$-system of the anthrax disease model by utilizing the Picard-Lindelof technique. By obtaining the basic reproduction number $\mathcal{R}_{0}$ of the fractional $\mathcal{C F}$-system we compute two disease-free and endemic equilibrium points and check the asymptotic stability property. Moreover, by applying an iterative approach based on the Sumudu transform we investigate the stability of the fractional $\mathcal{C} \mathcal{F}$-system. We obtain approximate series solutions of this system by means of the homotopy analysis transform method, in which we invoke the linear Laplace transform. Finally, after the convergence analysis of the numerical method HATM, we present a numerical simulation of the $\mathcal{C} \mathcal{F}$-fractional anthrax disease model and review the dynamical behavior of the solutions of this $\mathcal{C} \mathcal{F}$-system during a time interval.
\end{abstract}

MSC: Primary 34A08; secondary 34A12

Keywords: Anthrax disease; Homotopy analysis method; Mathematical modeling; Numerical simulation; The Caputo-Fabrizio derivative

\section{Introduction}

Analysis and investigation of various mathematical models of different natural processes is an applied branch of mathematics in which the researchers study dynamics of desired systems by means of some logical and computational tools. In this way, new fractional operators play an important role in modeling such natural phenomena and processes. Recently, the Caputo-Fabrizio fractional operator is utilized by many authors to analyze the existing systems (see, e.g., [1-8]). Also, there are many works on applications of fractional calculus (see, e.g., [9-26]). We apply this new fractional operator in the present research paper and recall its properties in the sequel.

Anthrax is considered as an infectious disease caused by the Bacillus Anthracis bacterium. Anthrax disease is categorized under zoonotic diseases and affects both animal

(c) The Author(s) 2020. This article is licensed under a Creative Commons Attribution 4.0 International License, which permits use sharing, adaptation, distribution and reproduction in any medium or format, as long as you give appropriate credit to the original author(s) and the source, provide a link to the Creative Commons licence, and indicate if changes were made. The images or other third party material in this article are included in the article's Creative Commons licence, unless indicated otherwise in a credit line to the material. If material is not included in the article's Creative Commons licence and your intended use is not permitted by statutory regulation or exceeds the permitted use, you will need to obtain permission directly from the copyright holder. To view a copy of this licence, visit http://creativecommons.org/licenses/by/4.0/. 
and human population [27]. Naturally, the anthrax disease can be found in soil and mostly has influence on herbivores as compared to carnivores [28]. This disease is one of the most dangerous infectious diseases in the world causing a vast and uncontrolled mortality in some animal populations such as pigs, sheep, horses, goats, cattle [29, 30]. According to Gutting et al. [31], this group of animals gets infected with Bacillus Anthracis bacterium through several ways including the consumption of infected water or grass, the inhalation of its spores, or contact with infected animals. Note that carcasses of infective animals can also pollute the environment. Grass and soil are the most important reservoirs of anthrax spores, which can cause the transmission of this disease between animals, because anthrax spores persist in the soil or grass for a long time under very extreme weather conditions. Also, the clinical symptoms of anthrax disease in infective animals take time to manifest since the incubation period of this disease is about three to eight days before these animals succumb to death.

The first simple model for dynamics of transmission of anthrax disease is formulated by Mushayabasa [32] in 2015. In this model the author regards three compartments entitled Susceptible, Contamination, and Pathogens. Mushayabasa does not discuss the role of infective animals in his model as a key factor in the transmission of anthrax infectious disease. One year later, Zerihun et al. [33] extended the Mushayabasa model and designed a new model of anthrax disease supplemented with four compartments entitled Susceptible, Contamination, Infective, and Pathogens. The compartment "Infective animals" has a key importance in this model, in which the clinical symptoms of anthrax transmit to susceptible animals [33]. After aforementioned works, some authors also studied various models of anthrax disease furnished with different compartments (see [34-36]).

For the proposed model of anthrax disease, in the present research, we are motivates by a research paper of Kimathi et al. [37], in which the usefulness of vaccination policy on SIR model is regarded in the context of a novel fractional modeling. In fact, the novelty of this work is that the compartment "Vaccinated animals" is added to the existing SIR model, and we generalize the classical system to a new fractional system based on a new fractional operator without singular kernel named the Caputo-Fabrizio derivative for the first time. We observe that the obtained approximate solutions of the fractional $\mathcal{C F}$-model of anthrax disease approach those of the classical integer-order system by passing the time.

More precisely, the contents of the paper is as follows. In the first step, we derive an existence criterion of solutions for the proposed fractional $\mathcal{C F}$-system of the anthrax disease model by utilizing the Picard-Lindelof technique. Then by obtaining the basic reproduction number $\mathcal{R}_{0}$ of the fractional $\mathcal{C F}$-system we compute two disease-free and endemic equilibrium points and check the asymptotic stability property. Moreover, by applying an iterative approach based on the Sumudu transform we investigate the stability of the fractional $\mathcal{C} \mathcal{F}$-system. We obtain the approximate series solutions of this system by means of the homotopy analysis transform method, in which we invoke the linear Laplace transform [38-40]. Finally, after the convergence analysis of the numerical method HATM, we present a numerical simulation of the $\mathcal{C F}$-fractional anthrax disease model and review the dynamical behavior of the solutions of this $\mathcal{C F}$-system during a time interval.

\section{Preliminaries}

In this part, we review some auxiliary and primitive concepts on the fractional operators. Assume that $\varrho \in(n-1, n]$ so that $n=[\varrho]+1$. For a function $\breve{w} \in \mathcal{A C}_{\mathbb{R}}^{(n)}([0,+\infty))$, the frac- 
tional derivative of Caputo type is given by

$$
{ }^{\mathcal{C}} \mathcal{D}_{0}^{\varrho} \breve{w}(t)=\int_{0}^{t} \frac{(t-z)^{n-\varrho-1}}{\Gamma(n-\varrho)} \breve{w}^{(n)}(z) \mathrm{d} z,
$$

provided that the integral is finite-valued [41, 42]. After that, a new fractional operator with no singular kernel is introduced by two Italian mathematicians Caputo and Fabrizio [43]. They assume that $a<b$ and $\breve{w} \in \mathcal{H}^{1}(a, b)$. Then the Caputo-Fabrizio or $(\mathcal{F C})$ derivative of order $\varrho \in(0,1]$ for a function $\breve{w}$ is given by

$$
{ }^{\mathcal{C F}} \mathcal{D}_{a}^{\varrho} \breve{w}(t)=\frac{(2-\varrho) M(\varrho)}{2(1-\varrho)} \int_{a}^{t} \exp \left(\frac{-\varrho}{1-\varrho}(t-z)\right) \breve{w}^{\prime}(z) \mathrm{d} z \quad(t \geq 0)
$$

where $M(\varrho)$ is a normalization function depending on the order $\varrho$ with $M(0)=M(1)=1$ [43]. Further, for $n \geq 1$ and $\varrho \in(0,1]$, we have ${ }^{\mathcal{C F}} \mathcal{D}_{a}^{\varrho+n} \breve{w}(t)={ }^{\mathcal{C F}} \mathcal{D}_{a}^{\varrho}\left(\mathcal{D}^{n} \breve{w}(t)\right)$ [3]. In 2015, Losada and Nieto [44] obtained a new explicit formula for the function $M(\varrho)=\frac{2}{2-\varrho}$ for $\varrho \in(0,1]$. In this case the fractional $\mathcal{C F}$-derivative for $\breve{w}$ is represented by

$$
{ }^{\mathcal{C F}} \mathcal{D}_{0}^{\varrho} \breve{w}(t)=\frac{1}{1-\varrho} \int_{0}^{t} \exp \left(\frac{-\varrho}{1-\varrho}(t-z)\right) \breve{w}^{\prime}(z) \mathrm{d} z \quad(t \geq 0) .
$$

It is clear that for each $\varrho \in(0,1]$, the equality ${ }^{\mathcal{C F}} \mathcal{D}_{0}^{\varrho} \breve{w}(t)=0$ is equivalent to $\breve{w}(t)=c^{*}$, where $c^{*}$ is an arbitrary constant. Also, Losada and Nieto defined the fractional $\mathcal{C} \mathcal{F}$-integral of order $\varrho \in(0,1]$ for $\breve{w}$ as follows:

$$
{ }^{\mathcal{C F}} \mathcal{I}_{0}^{\varrho} \breve{w}(t)=\frac{2(1-\varrho)}{(2-\varrho) M(\varrho)} \breve{w}(t)+\frac{2 \varrho}{(2-\varrho) M(\varrho)} \int_{0}^{t} \breve{w}(z) \mathrm{d} z
$$

for $t>0$ [44]. In this direction the authors prove that the unique solution of the fractionalorder differential equation ${ }^{\mathcal{C F}} \mathcal{D}_{0}^{\varrho} \breve{w}(t)=\breve{h}(t)$ is obtained by

$$
\breve{w}(t)=\breve{w}(0)+\frac{2(1-\varrho)}{(2-\varrho) M(\varrho)}(\breve{h}(t)-\breve{h}(0))+\frac{2 \varrho}{(2-\varrho) M(\varrho)} \int_{0}^{t} \breve{h}(z) \mathrm{d} z
$$

for $t \geq 0$ ([44]). For $\varrho \in(0,1]$, the Laplace transform of the fractional $\mathcal{C F}$-derivative is defined by

$$
\mathbb{L}\left[{ }^{\mathcal{C F}} \mathcal{D}_{0}^{\varrho+n} \breve{w}(t)\right](s)=\frac{s^{n+1} \mathbb{L}[\breve{w}(t)]-s^{n} \breve{w}(0)-s^{n-1} \breve{w}^{\prime}(0)-\cdots-\breve{w}^{(n)}(0)}{s+\varrho(1-s)},
$$

where $n \geq 1$ and $M(\varrho)=1$ [44]. In particular, for $n=1$ and $n=0$, we have

$$
\left\{\begin{array}{l}
\mathbb{L}\left[{ }^{\mathcal{C F}} \mathcal{D}_{0}^{\varrho+1} \breve{w}(t)\right](s)=\frac{s^{2} \mathbb{L}[\breve{w}(t)]-s \breve{w}(0)-\breve{w}^{\prime}(0)}{s \varrho(1-s)} \\
\mathbb{L}\left[{ }^{\mathcal{C F}} \mathcal{D}_{0}^{\varrho} \breve{w}(t)\right](s)=\frac{s \mathbb{L}[(t)]]^{\prime}(0)}{s+\varrho(1-s)}
\end{array}\right.
$$

In the light of the classical definition of the Fourier integral, the Sumudu transform can be derived [45-47]. For this aim, construct the following set

$$
\mathbb{A}=\left\{\breve{w}: \exists \beta, c_{1}, c_{2} \geq 0 \text { such that }|\breve{w}(t)|<\beta \exp \left(\frac{t}{c_{j}}\right), t \in(-1)^{j} \times[0, \infty)\right\} .
$$


Then the Sumudu transform of a function $\breve{w}(t) \in \mathbb{A}$ is represented by $\mathbb{S T}[\breve{w}(t)](s)=\breve{W}(s)$ and is defined as

$$
\breve{W}(s)=\mathbb{S T}[\breve{w}(t)](s)=\frac{1}{s} \int_{0}^{\infty} \exp \left(\frac{-t}{s}\right) \breve{w}(t) \mathrm{d} t \quad\left(s \in\left(-c_{1}, c_{2}\right)\right)
$$

for $t \geq 0$, and the inverse Sumudu transform of $\breve{W}(s)$ is denoted by $\breve{w}(t)=\mathbb{S T}^{-1}[\breve{W}(s)]$ [47]. Moreover, the Sumudu transform of the fractional derivative of the Caputo type is given by

$$
\mathbb{S T}\left[{ }^{\mathcal{C}} \mathcal{D}_{0}^{\varrho} \breve{w}(t)\right](s)=\frac{1}{s^{\varrho}}\left[\breve{W}(s)-\sum_{j=0}^{n} s^{\varrho-j}\left[{ }^{\mathcal{C}} \mathcal{D}_{0}^{\varrho-j} \breve{w}(t)\right]_{t=0}\right],
$$

where $n-1<\varrho \leq n$ [46]. Now assume that $\breve{w}$ is a function such that its $\mathcal{C F}$-derivative of fractional order exists. The Sumudu transform of the fractional $\mathcal{C F}$-derivative for $\breve{w}$ is defined by

$$
\mathbb{S T}\left[{ }^{\mathcal{C F}} \mathcal{D}_{0}^{\varrho} \breve{w}(t)\right](s)=\frac{M(\varrho)}{1-\varrho+\varrho s}(\mathbb{S T}[\breve{w}(t)](s)-\breve{w}(0))
$$

for $t \geq 0$ [48]. In the following, we review some notions about the stability. Let $(\mathbb{W}, d)$ be a metric space. We say that a self-map $\Psi: \mathbb{W} \rightarrow \mathbb{W}$ is the Picard operator if there is $p^{*} \in \mathbb{W}$ such that $\mathcal{F} \mathcal{I X}(\Psi)=\left\{p^{*}\right\}$ and, consequently, the convergent sequence $\left\{\Psi^{n}(p)\right\}_{n \in \mathbb{N}}$ tends to $p^{*}$ for all $p \in \mathbb{W}[49]$.

In this position, let us assume that $(\mathbb{W},\|\cdot\|)$ is a Banach space and $\Psi: \mathbb{W} \rightarrow \mathbb{W}$ is a selfmap on $\mathbb{W}$. Suppose that $\mathcal{F} \mathcal{I} \mathcal{X}(\Psi)=\{p \in \mathbb{W}: \Psi(p)=p\} \neq \emptyset$ is the collection of all fixed points of $\Psi$. Moreover, let $\left\{P_{n}\right\}_{n \geq 0} \subset \mathbb{W}$ be a sequence generated by the Picard iteration as follows: $P_{n+1}=\varphi\left(\Psi, P_{n}\right)(n=0,1,2, \ldots)$, where $P_{0} \in \mathbb{W}$ is the initial approximation, $\varphi$ is some function, and also $\lim _{n \rightarrow \infty} P_{n}=p \in \mathcal{F} \mathcal{I} \mathcal{X}(\Psi)$. Suppose that $\left\{\breve{f}_{n}\right\}_{n \geq 0} \subset \mathbb{W}$ and put

$$
\varepsilon_{n}=\left\|\breve{f}_{n+1}-\varphi\left(\Psi, \breve{f}_{n}\right)\right\| \quad(n=0,1,2, \ldots) .
$$

Then the recursive algorithm $P_{n+1}=\varphi\left(\Psi, P_{n}\right)$ is said to be Picard $\Psi$-stable with respect to $\Psi$ if and only if $\lim _{n \rightarrow \infty} \varepsilon_{n}=0$ implies that $\lim _{n \rightarrow \infty} \breve{f}_{n}=p$ [49].

Remark 2.1 ([49]) Note that if the sequence $\left\{\breve{f}_{n}\right\}$ has an upper bound, then $P_{n+1}=\Psi P_{n}$ is Picard $\Psi$-Stable whenever the Picard iteration $P_{n+1}=\Psi P_{n}$ satisfies all above assumptions.

The following theorem is utilized to prove the stability of the proposed fractional anthrax disease model.

Theorem $2.2([49])$ Suppose that $(\mathbb{W},\|\cdot\|)$ is a Banach space and $\Psi$ is a self-map on $\mathbb{W}$ satisfying the inequality

$$
\left\|\Psi_{p}-\Psi_{p^{\prime}}\right\| \leq K\left\|p-\Psi_{p}\right\|+k\left\|p-p^{\prime}\right\|
$$

for all $p, p^{\prime} \in \mathbb{W}$, where $K \geq 0$ and $0 \leq k<1$. Then $\Psi$ is Picard $\Psi$-Stable. 
We further derive an important criterion to confirm the asymptotic stability of a fractional linear system of the Caputo-Fabrizio type at free equilibrium point.

Proposition 2.3 ([50]) Let $\breve{w}(t) \in \mathbb{R}^{n}$ and $M \in \mathbb{R}^{n \times n}$. Then the characteristic equation related to the linear system

$$
{ }^{\mathcal{C F}} \mathcal{D}_{0}^{\varrho} \breve{w}(t)=M \breve{w}(t)
$$

supplemented with the Caputo-Fabrizio derivative of order $\varrho \in(0,1)$ is given by

$$
\operatorname{det}\left[s\left(I_{n \times n}-(1-\varrho) M\right)-\varrho M\right]=0 .
$$

Theorem 2.4 ([50]) Suppose that the matrix $\left(I_{n \times n}-(1-\varrho) M\right)$ is invertible. Then the fractional linear $\mathcal{C F}$-system (3) has the asymptotic stability property at a free equilibrium point if and only if all roots of the characteristic equation (4) for $\mathcal{C} \mathcal{F}$-system (3) have negative real parts.

\section{Fractional mathematical model of the anthrax disease}

In this section, we introduce a new fractional model of the anthrax disease in animals by applying a novel fractional operator with no singular kernel. In view of the implemented study by Kimathi and Wainaina [37], the classical first-order SIRV model of the anthrax disease in animals is formulated by the following four nonlinear differential equations:

$$
\left\{\begin{array}{l}
\frac{\mathrm{d} S}{\mathrm{~d} t}=\omega-\delta S(t) I(t)-(\rho+v) S(t)+\zeta R(t)+\varpi V(t), \\
\frac{\mathrm{d} I}{\mathrm{~d} t}=\delta S(t) I(t)-(\rho+\tau+\kappa) I(t), \\
\frac{\mathrm{d} R}{\mathrm{~d} t}=\kappa I(t)-(\rho+\zeta) R(t), \\
\frac{\mathrm{d} V}{\mathrm{~d} t}=v S(t)-(\rho+\varpi) V(t),
\end{array}\right.
$$

supplemented with initial conditions $S(0)=\breve{S}_{0}, I(0)=\breve{I}_{0}, R(0)=\breve{R}_{0}$, and $V(0)=\breve{V}_{0}$ [37]. Although human contribution in the transmission of the anthrax disease between animals is negligible, it becomes a very important subject of discussing the transmission of this disease in the animal population only. The fractional-order system (FDE) is related to systems with memory, history, or nonlocal effects, which exist in many biological systems that show the realistic biphasic decline behavior of infection or diseases but at a slower rate. In this model, since the internal memory effects of the biological system of the anthrax infection are not included, it is better that we extend the proposed ordinary model to a new fractional model. This shows that the fractional model of this animal disease yields the approximate results similar to the classical integer-order model. To modify the existing model, we convert the first-order ordinary derivative into the $\mathcal{C F}$-derivative of fractional order $\varrho \in(0,1]$ as follows:

$$
\left\{\begin{array}{l}
{ }^{\mathcal{F}} \mathcal{D}_{0}^{\varrho} S(t)=\omega-\delta S(t) I(t)-(\rho+v) S(t)+\zeta R(t)+\varpi V(t), \\
{ }^{\mathcal{F}} \mathcal{D}_{0}^{\varrho} I(t)=\delta S(t) I(t)-(\rho+\tau+\kappa) I(t), \\
{ }^{\mathcal{F}} \mathcal{D}_{0}^{\varrho} R(t)=\kappa I(t)-(\rho+\zeta) R(t), \\
{ }^{\mathcal{C F}} \mathcal{D}_{0}^{\varrho} V(t)=v S(t)-(\rho+\varpi) V(t),
\end{array}\right.
$$


furnished with initial conditions $S(0)=\breve{S}_{0}, I(0)=\breve{I}_{0}, R(0)=\breve{R}_{0}$, and $V(0)=\breve{V}_{0}$. In this mathematical framework, $S(t)$ represents the number of animals at risk of the anthrax infection at time $t$ (Susceptible), $I(t)$ indicates the number of animals with symptoms of this disease at time $t$ (Infected), $R(t)$ stands for the number of recovered animals from the anthrax infection and acquired temporal immunity at time $t$ (Recovered), and $V(t)$ denotes the number of vaccinated animals against attacks of mentioned anthrax disease at time $t$ (Vaccinated). In this case, it is obvious that the total number of animals included in these four classes at time $t$ equals $N(t)=S(t)+I(t)+R(t)+V(t)$.

Moreover, this new fractional model includes eight nonnegative parameters. The parameter $\omega$ denotes the recruitment rate, $\delta$ shows the contact rate, $\rho$ indicates the natural death rate, $v$ represents the vaccinated rate, $\zeta$ is the waning recovery rate, $\varpi$ stands for the waning immunity rate of vaccinated animals, $\tau$ indicates the disease-induced death rate, and the parameter $\kappa$ represents the recovery rate of animals. Besides, we need to notice that in the first-order ordinary system (5) of the disease model, the right-hand sides of four equations have dimensions $(\text { time })^{-1}$, but when we convert an integer order of these equations into the fractional order $\varrho$, the dimensions of the left-hand sides of four equations equal (time $)^{-} \varrho$. To match the dimensions of both sides of these differential equations, we have to change the dimensions of all nonnegative parameters $\omega, \delta, \rho, v, \zeta, \varpi, \tau$, and $\kappa$. In this position the modified version of the fractional system of the anthrax disease model formulated by (6) is as follows:

$$
\left\{\begin{array}{l}
{ }^{\mathcal{C}} \mathcal{D}_{0}^{\varrho} S(t)=\omega^{\varrho}-\delta^{\varrho} S(t) I(t)-\left(\rho^{\varrho}+v^{\varrho}\right) S(t)+\zeta^{\varrho} R(t)+\varpi^{\varrho} V(t) \\
{ }^{\mathcal{F}} \mathcal{D}_{0}^{\varrho} I(t)=\delta^{\varrho} S(t) I(t)-\left(\rho^{\varrho}+\tau^{\varrho}+\kappa^{\varrho}\right) I(t), \\
{ }^{\mathcal{C}} \mathcal{D}_{0}^{\varrho} R(t)=\kappa^{\varrho} I(t)-\left(\rho^{\varrho}+\zeta^{\varrho}\right) R(t) \\
{ }^{\mathcal{C}} \mathcal{D}_{0}^{\varrho} V(t)=v^{\varrho} S(t)-\left(\rho^{\varrho}+\varpi^{\varrho}\right) V(t) .
\end{array}\right.
$$

Numerical solutions of the modified fractional model (7) are obtained by utilizing the homotopy analysis transform method (HATM). To do this, the fractional differential equations of the above model are converted into algebraic equations by means of the Laplace transform. In the next section, we first derive an existence criterion of solutions for the fractional system (7).

\section{The existence criterion by Picard-Lindelof technique}

Hereafter, we consider the following fractional model of the anthrax disease by employing the Caputo-Fabrizio derivative:

$$
\left\{\begin{array}{l}
{ }^{\mathcal{C}} \mathcal{D}_{0}^{\varrho} S(t)=\omega^{\varrho}-\delta^{\varrho} S(t) I(t)-\left(\rho^{\varrho}+v^{\varrho}\right) S(t)+\zeta^{\varrho} R(t)+\varpi^{\varrho} V(t), \\
{ }^{\mathcal{C}} \mathcal{D}_{0}^{\varrho} I(t)=\delta^{\varrho} S(t) I(t)-\left(\rho^{\varrho}+\tau^{\varrho}+\kappa^{\varrho}\right) I(t), \\
{ }^{\mathcal{C} \mathcal{F}} \mathcal{D}_{0}^{\varrho} R(t)=\kappa^{\varrho} I(t)-\left(\rho^{\varrho}+\zeta^{\varrho}\right) R(t), \\
{ }^{\mathcal{C F}} \mathcal{D}_{0}^{\varrho} V(t)=v^{\varrho} S(t)-\left(\rho^{\varrho}+\varpi^{\varrho}\right) V(t),
\end{array}\right.
$$

furnished with initial conditions $S(0)=\breve{S}_{0}, I(0)=\breve{I}_{0}, R(0)=\breve{R}_{0}$, and $V(0)=\breve{V}_{0}$. To check the existence of solutions for the modified fractional system (8) of the anthrax disease model, we utilize the Picard-Lindelof technique. To do this, we first need to convert the anthrax 
disease model (8) into a fractional integral equation. In other words, we apply the fractional $\mathcal{C F}$-integral operator defined by Losada and Nieto [44] to both sides of differential equations (8). Then taking into account $(S(0), I(0), R(0), V(0))=\left(\breve{S}_{0}, \breve{I}_{0}, \breve{R}_{0}, \breve{V}_{0}\right)$, we have

$$
\begin{aligned}
S(t)= & \breve{S}_{0} \\
& +\frac{2(1-\varrho)}{(2-\varrho) M(\varrho)}\left(\omega^{\varrho}-\delta^{\varrho} S(t) I(t)-\left(\rho^{\varrho}+v^{\varrho}\right) S(t)+\zeta^{\varrho} R(t)+\varpi^{\varrho} V(t)\right) \\
& +\frac{2 \varrho}{(2-\varrho) M(\varrho)} \int_{0}^{t}\left[\omega^{\varrho}-\delta^{\varrho} S(z) I(z)-\left(\rho^{\varrho}+v^{\varrho}\right) S(z)+\zeta^{\varrho} R(z)+\varpi^{\varrho} V(z)\right] \mathrm{d} z \\
I(t)= & \breve{I}_{0}+\frac{2(1-\varrho)}{(2-\varrho) M(\varrho)}\left(\delta^{\varrho} S(t) I(t)-\left(\rho^{\varrho}+\tau^{\varrho}+\kappa^{\varrho}\right) I(t)\right) \\
& +\frac{2 \varrho}{(2-\varrho) M(\varrho)} \int_{0}^{t}\left[\delta^{\varrho} S(z) I(z)-\left(\rho^{\varrho}+\tau^{\varrho}+\kappa^{\varrho}\right) I(z)\right] \mathrm{d} z \\
R(t)= & \breve{R}_{0}+\frac{2(1-\varrho)}{(2-\varrho) M(\varrho)}\left(\kappa^{\varrho} I(t)-\left(\rho^{\varrho}+\zeta^{\varrho}\right) R(t)\right) \\
& +\frac{2 \varrho}{(2-\varrho) M(\varrho)} \int_{0}^{t}\left[\kappa^{\varrho} I(z)-\left(\rho^{\varrho}+\zeta^{\varrho}\right) R(z)\right] \mathrm{d} z \\
V(t)= & \breve{V}_{0}+\frac{2(1-\varrho)}{(2-\varrho) M(\varrho)}\left(v^{\varrho} S(t)-\left(\rho^{\varrho}+\varpi^{\varrho}\right) V(t)\right) \\
& +\frac{2 \varrho}{(2-\varrho) M(\varrho)} \int_{0}^{t}\left[v^{\varrho} S(z)-\left(\rho^{\varrho}+\varpi^{\varrho}\right) V(z)\right] \mathrm{d} z
\end{aligned}
$$

Now, due to (9)-(12), we define the Picard iterative algorithm as follows $(n=0,1,2, \ldots)$ :

$$
S_{0}(t)=\breve{S}_{0}, \quad I_{0}(t)=\breve{I}_{0}, \quad R_{0}(t)=\breve{R}_{0}, \quad V_{0}(t)=\breve{V}_{0},
$$

and

$$
\begin{aligned}
S_{n+1}(t)= & \frac{2(1-\varrho)}{(2-\varrho) M(\varrho)} \\
& \times\left(\omega^{\varrho}-\delta^{\varrho} S_{n}(t) I_{n}(t)-\left(\rho^{\varrho}+v^{\varrho}\right) S_{n}(t)+\zeta^{\varrho} R_{n}(t)+\varpi^{\varrho} V_{n}(t)\right) \\
& +\frac{2 \varrho}{(2-\varrho) M(\varrho)} \\
& \times \int_{0}^{t}\left[\omega^{\varrho}-\delta^{\varrho} S_{n}(z) I_{n}(z)-\left(\rho^{\varrho}+v^{\varrho}\right) S_{n}(z)+\zeta^{\varrho} R_{n}(z)+\varpi^{\varrho} V_{n}(z)\right] \mathrm{d} z, \\
I_{n+1}(t)= & \frac{2(1-\varrho)}{(2-\varrho) M(\varrho)}\left(\delta^{\varrho} S_{n}(t) I_{n}(t)-\left(\rho^{\varrho}+\tau^{\varrho}+\kappa^{\varrho}\right) I_{n}(t)\right) \\
& +\frac{2 \varrho}{(2-\varrho) M(\varrho)} \int_{0}^{t}\left[\delta^{\varrho} S_{n}(z) I_{n}(z)-\left(\rho^{\varrho}+\tau^{\varrho}+\kappa^{\varrho}\right) I_{n}(z)\right] \mathrm{d} z, \\
R_{n+1}(t)= & \frac{2(1-\varrho)}{(2-\varrho) M(\varrho)}\left(\kappa^{\varrho} I_{n}(t)-\left(\rho^{\varrho}+\zeta^{\varrho}\right) R_{n}(t)\right) \\
& +\frac{2 \varrho}{(2-\varrho) M(\varrho)} \int_{0}^{t}\left[\kappa^{\varrho} I_{n}(z)-\left(\rho^{\varrho}+\zeta^{\varrho}\right) R_{n}(z)\right] \mathrm{d} z,
\end{aligned}
$$




$$
\begin{aligned}
V_{n+1}(t)= & \frac{2(1-\varrho)}{(2-\varrho) M(\varrho)}\left(v^{\varrho} S_{n}(t)-\left(\rho^{\varrho}+\varpi^{\varrho}\right) V_{n}(t)\right) \\
& +\frac{2 \varrho}{(2-\varrho) M(\varrho)} \int_{0}^{t}\left[v^{\varrho} S_{n}(z)-\left(\rho^{\varrho}+\varpi^{\varrho}\right) V_{n}(z)\right] \mathrm{d} z .
\end{aligned}
$$

Now we assume that we can obtain the exact solutions of the fractional system (8) by taking the limits of both sides of (14)-(17) as $n$ tends to infinity. In other words, the solutions are obtained as follows:

$$
\left\{\begin{array}{l}
\lim _{n \rightarrow \infty} S_{n}(t)=S(t) \\
\lim _{n \rightarrow \infty} I_{n}(t)=I(t) \\
\lim _{n \rightarrow \infty} R_{n}(t)=R(t) \\
\lim _{n \rightarrow \infty} V_{n}(t)=V(t)
\end{array}\right.
$$

Here we are ready to derive the existence criterion and the uniqueness of the solutions based on the Picard-Lindelof approach. To reach this goal, define the following operators:

$$
\left\{\begin{array}{l}
\Upsilon_{1}(t, S):=\omega^{\varrho}-\delta^{\varrho} S(t) I(t)-\left(\rho^{\varrho}+v^{\varrho}\right) S(t)+\zeta^{\varrho} R(t)+\varpi^{\varrho} V(t), \\
\Upsilon_{2}(t, I):=\delta^{\varrho} S(t) I(t)-\left(\rho^{\varrho}+\tau^{\varrho}+\kappa^{\varrho}\right) I(t), \\
\Upsilon_{3}(t, R):=\kappa^{\varrho} I(t)-\left(\rho^{\varrho}+\zeta^{\varrho}\right) R(t), \\
\Upsilon_{4}(t, V):=v^{\varrho} S(t)-\left(\rho^{\varrho}+\varpi^{\varrho}\right) V(t),
\end{array}\right.
$$

where $\Upsilon_{1}(t, S), \Upsilon_{2}(t, I), \Upsilon_{3}(t, R)$, and $\Upsilon_{4}(t, V)$ are contractions with respect to $S, I, R$, and $V$ for the first, second, third, and fourth functions, respectively. Furthermore, we consider the following product spaces:

$$
\left\{\begin{array}{l}
\mathbb{I}_{a, b_{1}}:=[t-a, t+a] \times\left[S-b_{1}, S+b_{1}\right]=A \times B_{1}, \\
\mathbb{I}_{a, b_{2}}:=[t-a, t+a] \times\left[I-b_{2}, I+b_{2}\right]=A \times B_{2}, \\
\mathbb{I}_{a, b_{3}}:=[t-a, t+a] \times\left[R-b_{3}, R+b_{3}\right]=A \times B_{3}, \\
\mathbb{I}_{a, b_{4}}:=[t-a, t+a] \times\left[V-b_{4}, V+b_{4}\right]=A \times B_{4} .
\end{array}\right.
$$

Take

$$
\begin{aligned}
& \Upsilon_{1}^{*}=\sup _{(t, S) \in \mathbb{I}_{a, b_{1}}}\left\|\Upsilon_{1}(t, S(t))\right\|, \quad \Upsilon_{2}^{*}=\sup _{(t, I) \in \mathbb{I}_{a, b_{2}}}\left\|\Upsilon_{2}(t, I(t))\right\|, \\
& \Upsilon_{3}^{*}=\sup _{(t, R) \in \mathbb{I}_{a, b_{3}}}\left\|\Upsilon_{3}(t, R(t))\right\|,
\end{aligned}
$$

and

$$
\Upsilon_{4}^{*}=\sup _{(t, V) \in \mathbb{I}_{a, b_{4}}}\left\|\Upsilon_{4}(t, V(t))\right\|
$$

In this position, we define the Picard operator

$$
\mathcal{O}: \mathcal{C}\left(A, B_{1}, B_{2}, B_{3}, B_{4}\right) \rightarrow \mathcal{C}\left(A, B_{1}, B_{2}, B_{3}, B_{4}\right)
$$


as follows:

$$
\mathcal{O}(\mathbb{W}(t))=\mathbb{W}_{0}(t)+\frac{2(1-\varrho)}{(2-\varrho) M(\varrho)} \mathcal{G}(t, \mathbb{W}(t))+\frac{2 \varrho}{(2-\varrho) M(\varrho)} \int_{0}^{t} \mathcal{G}(z, \mathbb{W}(z)) \mathrm{d} z
$$

so that $\mathbb{W}(t)=\{S(t), I(t), R(t), V(t)\}, \mathbb{W}_{0}(t)=\left\{\breve{S}_{0}, \breve{I}_{0}, \breve{R}_{0}, \breve{V}_{0}\right\}$, and

$$
\mathcal{G}(t, \mathbb{W}(t))=\left\{\Upsilon_{1}(t, S(t)), \Upsilon_{2}(t, I(t)), \Upsilon_{3}(t, R(t)), \Upsilon_{4}(t, V(t))\right\}
$$

To apply the Picard theorem, we define the uniform norm on the space

$$
\mathcal{C}\left(A, B_{1}, B_{2}, B_{3}, B_{4}\right)
$$

as $\|\mathbb{W}\|_{\infty}=\sup _{t \in[t-a, t+a]=A}|\mathbb{W}(t)|$. In the following, we assume that all solution functions are bounded during a time interval, that is,

$$
\|\mathbb{W}\|_{\infty} \leq \max \left\{b_{1}, b_{2}, b_{3}, b_{4}\right\}=b .
$$

Moreover, let us assume that $\Upsilon^{*}=\max \left\{\Upsilon_{1}^{*}, \Upsilon_{2}^{*}, \Upsilon_{3}^{*}, \Upsilon_{4}^{*}\right\}$ and that there is $t_{0}$ with $t \leq t_{0}$. Then we have

$$
\begin{array}{rl}
\| \mathcal{O} & \mathbb{W}(t)-\mathbb{W}_{0}(t) \| \\
& =\left\|\frac{2(1-\varrho)}{(2-\varrho) M(\varrho)} \mathcal{G}(t, \mathbb{W}(t))+\frac{2 \varrho}{(2-\varrho) M(\varrho)} \int_{0}^{t} \mathcal{G}(z, \mathbb{W}(z)) \mathrm{d} z\right\| \\
& \leq \frac{2(1-\varrho)}{(2-\varrho) M(\varrho)}\|\mathcal{G}(t, \mathbb{W}(t))\|+\frac{2 \varrho}{(2-\varrho) M(\varrho)} \int_{0}^{t}\|\mathcal{G}(z, \mathbb{W}(z))\| \mathrm{d} z \\
& \leq\left[\frac{2(1-\varrho)}{(2-\varrho) M(\varrho)}+\frac{2 \varrho t_{0}}{(2-\varrho) M(\varrho)}\right] \Upsilon^{*}=\mu^{*} \Upsilon^{*} \leq b,
\end{array}
$$

where we assume that $\mu^{*}<\frac{b}{\Upsilon^{*}}$ and also $\mu^{*}=\frac{2(1-\varrho)}{(2-\varrho) M(\varrho)}+\frac{2 \varrho t_{0}}{(2-\varrho) M(\varrho)}$. Finally, we intend to show that the Picard operator $\mathcal{O}$ is a contraction. Since the functions $\Upsilon_{1}, \Upsilon_{2}, \Upsilon_{3}$, and $\Upsilon_{4}$ are contractions, for all $\mathbb{W}_{1}, \mathbb{W}_{2} \in \mathcal{C}\left(A, B_{1}, B_{2}, B_{3}, B_{4}\right)$, we can write

$$
\left\|\mathcal{G}\left(t, \mathbb{W}_{1}(t)\right)-\mathcal{G}\left(t, \mathbb{W}_{2}(t)\right)\right\| \leq \lambda^{*}\left\|\mathbb{W}_{1}(t)-\mathbb{W}_{2}(t)\right\|
$$

where $\lambda^{*}<1$ is the contraction constant. At this moment, using the definition of the Picard operator $\mathcal{O}$ given in (21), inequality (24), and the equality

$$
\left\|\mathcal{O} \mathbb{W}_{1}-\mathcal{O} \mathbb{W}_{1}\right\|=\sup _{t \in A}\left|\mathbb{W}_{1}(t)-\mathbb{W}_{2}(t)\right|
$$

we get

$$
\begin{aligned}
\left\|\mathcal{O}\left(\mathbb{W}_{1}(t)\right)-\mathcal{O}\left(\mathbb{W}_{2}(t)\right)\right\|= & \| \frac{2(1-\varrho)}{(2-\varrho) M(\varrho)}\left[\mathcal{G}\left(t, \mathbb{W}_{1}(t)\right)-\mathcal{G}\left(t, \mathbb{W}_{2}(t)\right)\right] \\
& +\frac{2 \varrho}{(2-\varrho) M(\varrho)} \int_{0}^{t}\left[\mathcal{G}\left(z, \mathbb{W}_{1}(z)\right)-\mathcal{G}\left(z, \mathbb{W}_{2}(z)\right)\right] \mathrm{d} z \|
\end{aligned}
$$




$$
\begin{aligned}
\leq & \frac{2(1-\varrho)}{(2-\varrho) M(\varrho)}\left\|\mathcal{G}\left(t, \mathbb{W}_{1}(t)\right)-\mathcal{G}\left(t, \mathbb{W}_{2}(t)\right)\right\| \\
& +\frac{2 \varrho}{(2-\varrho) M(\varrho)} \int_{0}^{t}\left\|\mathcal{G}\left(z, \mathbb{W}_{1}(z)\right)-\mathcal{G}\left(z, \mathbb{W}_{2}(z)\right)\right\| \mathrm{d} z \\
\leq & \frac{2(1-\varrho) \lambda^{*}}{(2-\varrho) M(\varrho)}\left\|\mathbb{W}_{1}(t)-\mathbb{W}_{2}(t)\right\| \\
& +\frac{2 \varrho \lambda^{*}}{(2-\varrho) M(\varrho)} \int_{0}^{t}\left\|\mathbb{W}_{1}(z)-\mathbb{W}_{2}(z)\right\| \mathrm{d} z \\
\leq & {\left[\frac{2(1-\varrho)}{(2-\varrho) M(\varrho)}+\frac{2 \varrho t_{0}}{(2-\varrho) M(\varrho)}\right] \lambda^{*}\left\|\mathbb{W}_{1}(t)-\mathbb{W}_{2}(t)\right\| } \\
= & \mu^{*} \lambda^{*}\left\|\mathbb{W}_{1}(t)-\mathbb{W}_{2}(t)\right\| .
\end{aligned}
$$

Thus we obtain

$$
\left\|\mathcal{O} \mathbb{W}_{1}-\mathcal{O} \mathbb{W}_{2}\right\|_{\infty} \leq \mu^{*} \lambda^{*}\left\|\mathbb{W}_{1}-\mathbb{W}_{2}\right\|_{\infty}
$$

which indicates that the operator $\mathcal{O}$ is a contraction with constant $\mu^{*} \lambda^{*}<1$ since $\lambda^{*}<1$. Hence the Banach fixed point theorem implies that the fractional system (8) of the anthrax disease model has a unique solution.

\section{Equilibrium points of the fractional $\mathcal{C F}$-model (8)}

In this section, we intend to obtain the equilibrium points of the fractional anthrax disease $\mathcal{C F}$-model (8). For this aim, we first solve the following homogeneous equations:

$$
{ }^{\mathcal{C} F} \mathcal{D}_{0}^{\varrho} S(t)={ }^{\mathcal{C F}} \mathcal{D}_{0}^{\varrho} I(t)={ }^{\mathcal{C F}} \mathcal{D}_{0}^{\varrho} R(t)={ }^{\mathcal{C F}} \mathcal{D}_{0}^{\varrho} V(t)=0 .
$$

Consequently, a disease-free equilibrium point of the fractional $\mathcal{C F}$-system (8) is given by $\mathcal{E}^{0}=\left(S^{0}, I^{0}, R^{0}, V^{0}\right)$, where

$$
S^{0}=\frac{\left(\rho^{\varrho}+\varpi^{\varrho}\right) \omega^{\varrho}}{\rho^{\varrho}\left(\rho^{\varrho}+\varpi^{\varrho}+v^{\varrho}\right)}, \quad I^{0}=0, \quad R^{0}=0, \quad V^{0}=\frac{v^{\varrho} \omega^{\varrho}}{\rho^{\varrho}\left(\rho^{\varrho}+\varpi^{\varrho}+v^{\varrho}\right)} .
$$

To find the endemic equilibrium point for the fractional $\mathcal{C F}$-system (8), we need to determine a basic reproduction number $\mathcal{R}_{0}$. This quantity appears by applying the nextgeneration matrix process introduced by Van den Driessche [51]. To obtain the basic reproduction number $\mathcal{R}_{0}$, set

$$
A=\left[\begin{array}{c}
\delta^{\varrho} S I \\
0 \\
0 \\
0
\end{array}\right] \text { and } B=\left[\begin{array}{c}
\left(\rho^{\varrho}+\tau^{\varrho}+\kappa^{\varrho}\right) I \\
-\omega^{\varrho}+\delta^{\varrho} S I+\left(\rho^{\varrho}+v^{\varrho}\right) S-\zeta^{\varrho} R-\varpi^{\varrho} V \\
-\kappa^{\varrho} I+\left(\rho^{\varrho}+\zeta^{\varrho}\right) R \\
-v^{\varrho} S+\left(\rho^{\varrho}+\varpi^{\varrho}\right) V
\end{array}\right]
$$


Then the Jacobian matrices of both matrices $A$ and $B$ at disease-free equilibrium point $\mathcal{E}^{0}$ given in (26) are defined as follows:

$$
[\mathfrak{J}(A)]\left(\mathcal{E}^{0}\right)=\left[\begin{array}{cccc}
0 & \frac{\delta^{\varrho} \omega^{\varrho}\left(\rho^{\varrho}+\varpi^{\varrho}\right)}{\rho^{\varrho}\left(\rho^{\varrho}+\omega^{\varrho}+v^{\varrho}\right)} & 0 & 0 \\
0 & 0 & 0 & 0 \\
0 & 0 & 0 & 0 \\
0 & 0 & 0 & 0
\end{array}\right]
$$

and

$$
[\mathfrak{J}(B)]\left(\mathcal{E}^{0}\right)=\left[\begin{array}{cccc}
0 & \rho^{\varrho}+\tau^{\varrho}+\kappa^{\varrho} & 0 & 0 \\
0 & \frac{\delta^{\varrho} \omega^{\varrho}\left(\rho^{\varrho}+\varpi^{\varrho}\right)}{\rho^{\varrho}\left(\rho^{\varrho}+\varpi^{\varrho}+v^{\varrho}\right)} & -\zeta^{\varrho} & -\varpi^{\varrho} \\
0 & -\kappa^{\varrho} & \rho^{\varrho}+\zeta^{\varrho} & 0 \\
-v^{\varrho} & 0 & 0 & \rho^{\varrho}+\varpi^{\varrho}
\end{array}\right]
$$

In view of (27) and (28), by some routine computations we obtain

$$
[\mathfrak{J}(A)]\left(\mathcal{E}^{0}\right) \cdot[\mathfrak{J}(B)]^{-1}\left(\mathcal{E}^{0}\right)=\left[\begin{array}{cccc}
\frac{\delta^{\varrho} \omega^{\varrho}\left(\rho^{\varrho}+\varpi^{\varrho}\right)}{\rho^{\varrho}\left(\rho^{\varrho}+\tau^{\varrho}+\kappa^{\varrho}\right)\left(\rho^{\varrho}+\varpi^{\varrho}+v^{\varrho}\right)} & 0 & 0 & 0 \\
0 & 0 & 0 & 0 \\
0 & 0 & 0 & 0 \\
0 & 0 & 0 & 0
\end{array}\right] .
$$

In the final step, we find the eigenvalue of the characteristic equation

$$
\operatorname{det}\left[I_{4 \times 4}-\lambda\left([\mathfrak{J}(A)]\left(\mathcal{E}^{0}\right) \cdot[\mathfrak{J}(B)]^{-1}\left(\mathcal{E}^{0}\right)\right)\right]=0,
$$

and so the basic reproduction number $\mathcal{R}_{0}$ is obtained as follows:

$$
\mathcal{R}_{0}=\lambda=\frac{\rho^{\varrho}\left(\rho^{\varrho}+\tau^{\varrho}+\kappa^{\varrho}\right)\left(\rho^{\varrho}+\varpi^{\varrho}+v^{\varrho}\right)}{\delta^{\varrho} \omega^{\varrho}\left(\rho^{\varrho}+\varpi^{\varrho}\right)} .
$$

The basic reproduction number $\mathcal{R}_{0}$ is a metric to measure the transmission potential of a infectious disease over the time. When the value of $\mathcal{R}_{0}$ is greater than one, the fractional $\mathcal{C F}$-system (8) has an endemic equilibrium point $\mathcal{E}^{*}=\left(S^{*}, I^{*}, R^{*}, V^{*}\right)$. More precisely, to obtain an endemic equilibrium point $\mathcal{E}^{*}$, we have to solve equations (25) assuming that all variables $S(t), I(t), R(t)$, and $V(t)$ are nonzero. Equations (25) can be rewritten as follows:

$$
\begin{aligned}
& \omega^{\varrho}-\delta^{\varrho} S(t) I(t)-\left(\rho^{\varrho}+v^{\varrho}\right) S(t)+\zeta^{\varrho} R(t)+\varpi^{\varrho} V(t)=0, \\
& \delta^{\varrho} S(t) I(t)-\left(\rho^{\varrho}+\tau^{\varrho}+\kappa^{\varrho}\right) I(t)=0, \\
& \kappa^{\varrho} I(t)-\left(\rho^{\varrho}+\zeta^{\varrho}\right) R(t)=0, \\
& v^{\varrho} S(t)-\left(\rho^{\varrho}+\varpi^{\varrho}\right) V(t)=0 .
\end{aligned}
$$

From equation (31) we have $I(t)\left[\delta^{\varrho} S(t)-\left(\rho^{\varrho}+\tau^{\varrho}+\kappa^{\varrho}\right)\right]=0$. Since $I(t) \neq 0$, we can obtain $S^{*}(t)=\frac{\rho^{\varrho}+\tau^{\varrho}+\kappa^{\varrho}}{\delta^{\varrho}}$. Moreover, from equation (33) we have $V^{*}(t)=\frac{v^{\varrho}\left(\rho^{\varrho}+\tau^{\varrho}+\kappa^{\varrho}\right)}{\delta\left(\rho^{\varrho}+\sigma^{\varrho}\right)}$. Finally, if we combine equations (30) and (31), then by solving the obtained system we get

$$
R^{*}(t)=\frac{\kappa^{\varrho}\left[\omega^{\varrho} \delta^{\varrho}\left(\rho^{\varrho}+\varpi^{\varrho}\right)-\rho^{\varrho}\left(\rho^{\varrho}+\tau^{\varrho}+\kappa^{\varrho}\right)\left(\rho^{\varrho}+v^{\varrho}+\varpi^{\varrho}\right)\right]}{\delta^{\varrho}\left(\rho^{\varrho}+\varpi^{\varrho}\right)\left[\rho^{\varrho}\left(\rho^{\varrho}+\tau^{\varrho}+\kappa^{\varrho}+\zeta^{\varrho}\right)+\zeta^{\varrho} \tau^{\varrho}\right]}
$$


and

$$
I^{*}(t)=\frac{\left(\rho^{\varrho}+\zeta^{\varrho}\right)\left[\omega^{\varrho} \delta^{\varrho}\left(\rho^{\varrho}+\varpi^{\varrho}\right)-\rho^{\varrho}\left(\rho^{\varrho}+\tau^{\varrho}+\kappa^{\varrho}\right)\left(\rho^{\varrho}+v^{\varrho}+\varpi^{\varrho}\right)\right]}{\delta^{\varrho}\left(\rho^{\varrho}+\varpi^{\varrho}\right)\left[\rho^{\varrho}\left(\rho^{\varrho}+\tau^{\varrho}+\kappa^{\varrho}+\zeta^{\varrho}\right)+\zeta^{\varrho} \tau^{\varrho}\right]} .
$$

Hence the components of an endemic equilibrium point $\mathcal{E}^{*}=\left(S^{*}, I^{*}, R^{*}, V^{*}\right)$ for the fractional $\mathcal{C} \mathcal{F}$-system (8) are obtained as before.

In this position, we want to check the asymptotic stability property of the disease-free equilibrium point $\mathcal{E}^{0}$ obtained in (26) for the fractional $\mathcal{C} \mathcal{F}$-system (8) of the anthrax disease model. By some simple computations we get that the Jacobian matrix of the fractional $\mathcal{C F}$-system (8) at disease-free equilibrium point $\mathcal{E}^{0}$ is defined by

$$
\mathfrak{J}\left(\mathcal{E}^{0}\right)=\left[\begin{array}{cccc}
-\left(\rho^{\varrho}+v^{\varrho}\right) & -\frac{\delta^{\varrho} \omega^{\varrho}\left(\rho^{\varrho}+\varpi^{\varrho}\right)}{\rho^{\varrho}\left(\rho^{\varrho}+\varpi^{\varrho}+v^{\varrho}\right)} & \zeta^{\varrho} & \varpi^{\varrho} \\
0 & \frac{\delta^{\varrho} \omega^{\varrho}\left(\rho^{\varrho}+\varpi^{\varrho}\right)}{\rho^{\varrho}\left(\rho^{\varrho}+\varpi^{\varrho}+v^{\varrho}\right)}-\left(\rho^{\varrho}+\tau^{\varrho}+\kappa^{\varrho}\right) & 0 & 0 \\
0 & \kappa^{\varrho} & -\left(\rho^{\varrho}+\zeta^{\varrho}\right) & 0 \\
v^{\varrho} & 0 & 0 & -\left(\rho^{\varrho}+\varpi^{\varrho}\right)
\end{array}\right] .
$$

Hence the characteristic equation of the mentioned $\mathcal{C F}$-system (8) is given by

$$
\operatorname{det}\left[s\left(I_{4 \times 4}-(1-\varrho) \mathcal{J}\left(\mathcal{E}^{0}\right)\right)-\varrho \mathcal{J}\left(\mathcal{E}^{0}\right)\right]=0
$$

Then we can state the following theorem and confirm that the disease-free equilibrium point $\mathcal{E}^{0}$ of $\mathcal{C} \mathcal{F}$-system (8) is asymptotically stable.

Theorem 5.1 The disease-free equilibrium point $\mathcal{E}^{0}$ of the fractional $\mathcal{C} \mathcal{F}$-system of the anthrax disease model (8) has the asymptotic stability property whenever real parts of all roots of the characteristic equation (34) are negative.

Proof In view of the Jacobian matrix $\mathcal{J}\left(\mathcal{E}^{0}\right)$, applying the matrix equation (34), we obtain the characteristic equation of the fractional $\mathcal{C F}$-system (8)

$$
\begin{aligned}
{[s(1-} & \left.\left.(1-\varrho) P^{*}\right)-\varrho P^{*}\right]\left[s\left(1+(1-\varrho)\left(\rho^{\varrho}+\zeta^{\varrho}\right)\right)+\varrho\left(\rho^{\varrho}+\zeta^{\varrho}\right)\right] \\
& \times\left(\left[s\left(1+(1-\varrho)\left(\rho^{\varrho}+v^{\varrho}\right)\right)+\varrho\left(\rho^{\varrho}+v^{\varrho}\right)\right]\right. \\
& \times\left[s\left(1+(1-\varrho)\left(\rho^{\varrho}+\varpi^{\varrho}\right)\right)+\varrho\left(\rho^{\varrho}+\varpi^{\varrho}\right)\right] \\
& \left.\quad-\left[s(1-\varrho) v^{\varrho}-\varrho v^{\varrho}\right]\left[s(1-\varrho) \varpi^{\varrho}-\varrho \varpi^{\varrho}\right]\right) \\
= & 0,
\end{aligned}
$$

where $P^{*}=\frac{\delta^{\varrho} \omega^{\varrho}\left(\rho^{\varrho}+\varpi^{\varrho}\right)}{\rho^{\varrho}\left(\rho^{\varrho}+\varpi^{\varrho}+v^{\varrho}\right)}-\left(\rho^{\varrho}+\tau^{\varrho}+\kappa^{\varrho}\right)$. The eigenvalues of this characteristic equation are

$$
s_{1}=\frac{\varrho P^{*}}{1-(1-\varrho) P^{*}}, \quad s_{2}=\frac{-\varrho\left(\rho^{\varrho}+\zeta^{\varrho}\right)}{1+(1-\varrho)\left(\rho^{\varrho}+\zeta^{\varrho}\right)},
$$

and the roots of the equation $s^{2}+B^{*} s+C^{*}=0$ where

$$
B^{*}=\frac{\varrho\left(2 \rho^{\varrho}+\varpi^{\varrho}+v^{\varrho}\right)+2 \varrho(1-\varrho)\left[\rho^{2 \varrho}+\rho^{\varrho} \varpi^{\varrho}+\rho^{\varrho} v^{\varrho}+2 v^{\varrho} \varpi^{\varrho}\right]}{1+(1-\varrho)\left[\left(\rho^{\varrho}+\varpi^{\varrho}\right)\left(\rho^{\varrho}+v^{\varrho}\right)\right]+(1-\varrho)^{2}\left[\rho^{\varrho}\left(\rho^{\varrho}+\varpi^{\varrho}+v^{\varrho}\right)\right]}
$$


and

$$
C^{*}=\frac{\varrho^{2}\left[\rho^{\varrho}\left(\rho^{\varrho}+\varpi^{\varrho}+v^{\varrho}\right)\right]}{1+(1-\varrho)\left[\left(\rho^{\varrho}+\varpi^{\varrho}\right)\left(\rho^{\varrho}+v^{\varrho}\right)\right]+(1-\varrho)^{2}\left[\rho^{\varrho}\left(\rho^{\varrho}+\varpi^{\varrho}+v^{\varrho}\right)\right]} .
$$

If $(1-\varrho) P^{*}>1$, then since $\varrho \in(0,1], P^{*}>0$, and so $\frac{\delta^{\varrho} \omega^{\varrho}\left(\rho^{\varrho}+\varpi^{\varrho}\right)}{\rho^{\varrho}\left(\rho^{\varrho}+\varpi^{\varrho}+v^{\varrho}\right)}>\left(\rho^{\varrho}+\tau^{\varrho}+\kappa^{\varrho}\right)$. This means that $s_{1}$ is a root with negative sign. Also, as we said before, all parameters are positive, so it is clear that $s_{2}$ is negative. Moreover, the roots of equation $s^{2}+B^{*} s+C^{*}=0$ must also be negative. To reach this goal, since $\varrho \in(0,1], B^{*}>0$ and $C^{*}>0$, and thus by the RouthHurwitz criterion we find that all roots of the characteristic equation (35) are negative. Hence if $(1-\varrho) P^{*}>1$, then the disease-free equilibrium point $\mathcal{E}^{0}$ of the fractional $\mathcal{C} \mathcal{F}$ system of the anthrax disease model (8) has the asymptotic stability property, and the proof is completed.

\section{Stability analysis via iterative approach}

To analyze the stability of the fractional anthrax disease model (8), we provide an iterative formula by means of the Sumudu transform. For this aim, we get

$$
\left\{\begin{array}{l}
\mathbb{S T}\left[{ }^{\mathcal{C F}} \mathcal{D}_{0}^{\varrho} S(t)\right](s)=\mathbb{S T}\left[\omega^{\varrho}-\delta^{\varrho} S(t) I(t)-\left(\rho^{\varrho}+v^{\varrho}\right) S(t)+\zeta^{\varrho} R(t)+\varpi^{\varrho} V(t)\right](s), \\
\mathbb{S T}\left[{ }^{\mathcal{C F}} \mathcal{D}_{0}^{\varrho} I(t)\right](s)=\mathbb{S T}\left[\delta^{\varrho} S(t) I(t)-\left(\rho^{\varrho}+\tau^{\varrho}+\kappa^{\varrho}\right) I(t)\right](s), \\
\mathbb{S T}\left[{ }^{\mathcal{C F}} \mathcal{D}_{0}^{\varrho} R(t)\right](s)=\mathbb{S T}\left[\kappa^{\varrho} I(t)-\left(\rho^{\varrho}+\zeta^{\varrho}\right) R(t)\right](s), \\
\mathbb{S T}\left[{ }^{\mathcal{C F}} \mathcal{D}_{0}^{\varrho} V(t)\right](s)=\mathbb{S T}\left[v^{\varrho} S(t)-\left(\rho^{\varrho}+\varpi^{\varrho}\right) V(t)\right](s) .
\end{array}\right.
$$

By the definition of the Sumudu transform for the fractional $\mathcal{C F}$-derivative we obtain

$$
\left\{\begin{array}{l}
\frac{M(\varrho)}{1-\varrho+\varrho s}(\mathbb{S T}[S(t)](s)-S(0)) \\
=\mathbb{S} \mathbb{T}\left[\omega^{\varrho}-\delta^{\varrho} S(t) I(t)-\left(\rho^{\varrho}+v^{\varrho}\right) S(t)+\zeta^{\varrho} R(t)+\varpi^{\varrho} V(t)\right](s), \\
\frac{M(\varrho)}{1-\varrho+\varrho s}(\mathbb{S T}[I(t)](s)-I(0))=\mathbb{S T}\left[\delta^{\varrho} S(t) I(t)-\left(\rho^{\varrho}+\tau^{\varrho}+\kappa^{\varrho}\right) I(t)\right](s), \\
\frac{M(\varrho)}{1-\varrho+\varrho s}(\mathbb{S T}[R(t)](s)-R(0))=\mathbb{S T}\left[\kappa^{\varrho} I(t)-\left(\rho^{\varrho}+\zeta^{\varrho}\right) R(t)\right](s), \\
\frac{M(\varrho)}{1-\varrho+\varrho s}(\mathbb{S T}[V(t)](s)-V(0))=\mathbb{S T}\left[v^{\varrho} S(t)-\left(\rho^{\varrho}+\varpi^{\varrho}\right) V(t)\right](s) .
\end{array}\right.
$$

By rewriting the formulas we obtain the following equalities:

$$
\left\{\begin{array}{l}
\mathbb{S T}[S(t)](s) \\
\quad=S(0)+\frac{1-\varrho+\varrho s}{M(\varrho)} \mathbb{S T}\left[\omega^{\varrho}-\delta^{\varrho} S(t) I(t)-\left(\rho^{\varrho}+v^{\varrho}\right) S(t)+\zeta^{\varrho} R(t)+\varpi^{\varrho} V(t)\right](s), \\
\mathbb{S T}[I(t)](s)=I(0)+\frac{1-\varrho+\varrho s}{M(\varrho)} \mathbb{S T}\left[\delta^{\varrho} S(t) I(t)-\left(\rho^{\varrho}+\tau^{\varrho}+\kappa^{\varrho}\right) I(t)\right](s), \\
\mathbb{S T}[R(t)](s)=R(0)+\frac{1-\varrho+\varrho s}{M(\varrho)} \mathbb{S} \mathbb{T}\left[\kappa^{\varrho} I(t)-\left(\rho^{\varrho}+\zeta^{\varrho}\right) R(t)\right](s), \\
\mathbb{S T}[V(t)](s)=V(0)+\frac{1-\varrho+\varrho s}{M(\varrho)} \mathbb{S} \mathbb{T}\left[v^{\varrho} S(t)-\left(\rho^{\varrho}+\varpi^{\varrho}\right) V(t)\right](s) .
\end{array}\right.
$$


Now, after taking the inverse Sumudu transform on both sides of system (38), the we obtain the following recursive equations for the fractional $\mathcal{C F}$-model (8):

$$
\left\{\begin{array}{l}
S_{n+1}(t) \\
\quad=S_{n}(0)+\mathbb{S T}^{-1}\left[\frac{1-\varrho+\varrho s}{M(\varrho)} \mathbb{S T}\left[\omega^{\varrho}-\delta^{\varrho} S_{n}(t) I_{n}(t)-\left(\rho^{\varrho}+v^{\varrho}\right) S_{n}(t)+\zeta^{\varrho} R_{n}(t)+\varpi^{\varrho} V_{n}(t)\right](s)\right] \\
I_{n+1}(t)=I_{n}(0)+\mathbb{S T}^{-1}\left[\frac{1-\varrho+\varrho s}{M(\varrho)} \mathbb{S T}\left[\delta^{\varrho} S_{n}(t) I_{n}(t)-\left(\rho^{\varrho}+\tau^{\varrho}+\kappa^{\varrho}\right) I_{n}(t)\right](s)\right] \\
R_{n+1}(t)=R_{n}(0)+\mathbb{S T}^{-1}\left[\frac{1-\varrho+\varrho s}{M(\varrho)} \mathbb{S T}\left[\kappa^{\varrho} I_{n}(t)-\left(\rho^{\varrho}+\zeta^{\varrho}\right) R_{n}(t)\right](s)\right] \\
V_{n+1}(t)=V_{n}(0)+\mathbb{S T}^{-1}\left[\frac{1-\varrho+\varrho)}{M(\varrho)} \mathbb{S T}\left[v^{\varrho} S_{n}(t)-\left(\rho^{\varrho}+\varpi^{\varrho}\right) V_{n}(t)\right](s)\right]
\end{array}\right.
$$

On the other hand, we obtain the approximate solutions of this $\mathcal{C F}$-system by

$$
\begin{array}{ll}
S(t)=\lim _{n \rightarrow \infty} S_{n}(t), & I(t)=\lim _{n \rightarrow \infty} I_{n}(t), \\
R(t)=\lim _{n \rightarrow \infty} R_{n}(t), & V(t)=\lim _{n \rightarrow \infty} V_{n}(t) .
\end{array}
$$

Now we can check the stability of the fractional $\mathcal{C F}$-system by considering the above notions and relations.

Theorem 6.1 Suppose that $\Psi$ is a self-map defined as follows:

$$
\begin{aligned}
\Psi\left(S_{n}(t)\right)= & S_{n+1}(t)=S_{n}(t) \\
& +\mathbb{S T}^{-1}\left[\frac { 1 - \varrho + \varrho s } { M ( \varrho ) } \mathbb { S T } \left[\omega^{\varrho}-\delta^{\varrho} S_{n}(t) I_{n}(t)\right.\right. \\
& \left.\left.-\left(\rho^{\varrho}+v^{\varrho}\right) S_{n}(t)+\zeta^{\varrho} R_{n}(t)+\varpi^{\varrho} V_{n}(t)\right](s)\right], \\
\Psi\left(I_{n}(t)\right)= & I_{n+1}(t)=I_{n}(t) \\
& +\mathbb{S T}^{-1}\left[\frac{1-\varrho+\varrho s}{M(\varrho)} \mathbb{S T}\left[\delta^{\varrho} S_{n}(t) I_{n}(t)-\left(\rho^{\varrho}+\tau^{\varrho}+\kappa^{\varrho}\right) I_{n}(t)\right](s)\right], \\
\Psi\left(R_{n}(t)\right)= & R_{n+1}(t)=R_{n}(t) \\
& +\mathbb{S T}^{-1}\left[\frac{1-\varrho+\varrho s}{M(\varrho)} \mathbb{S T}\left[\kappa^{\varrho} I_{n}(t)-\left(\rho^{\varrho}+\zeta^{\varrho}\right) R_{n}(t)\right](s)\right], \\
\Psi\left(V_{n}(t)\right)= & V_{n+1}(t)=V_{n}(t) \\
& +\mathbb{S T}^{-1}\left[\frac{1-\varrho+\varrho s}{M(\varrho)} \mathbb{S T}\left[v^{\varrho} S_{n}(t)-\left(\rho^{\varrho}+\varpi^{\varrho}\right) V_{n}(t)\right](s)\right] .
\end{aligned}
$$

Then the iteration fractional $\mathcal{C} \mathcal{F}$-system (41) is $\Psi$-stable in $\mathcal{L}^{1}(a, b)$ whenever we have

$$
\left\{\begin{array}{l}
1-\delta^{\varrho} K_{1}^{*} \Phi_{1}(t)-\delta^{\varrho} K_{2}^{*} \Phi_{2}(t)-\left(\rho^{\varrho}+v^{\varrho}\right) \Phi_{3}(t)+\zeta^{\varrho} \Phi_{4}(t)+\varpi^{\varrho} \Phi_{5}(t)<1 \\
1+\delta^{\varrho} K_{1}^{*} \Phi_{6}(t)+\delta^{\varrho} K_{2}^{*} \Phi_{7}(t)-\left(\rho^{\varrho}+\tau^{\varrho}+\kappa^{\varrho}\right) \Phi_{8}(t)<1 \\
1+\kappa^{\varrho} \Phi_{9}(t)-\left(\rho^{\varrho}+\zeta^{\varrho}\right) \Phi_{10}(t)<1 \\
1+v^{\varrho} \Phi_{11}(t)-\left(\rho^{\varrho}+\varpi^{\varrho}\right) \Phi_{12}(t)<1
\end{array}\right.
$$

where the functions $\Phi_{j}, j=1,2, \ldots, 12$, are introduced further. 
Proof To begin the proof, we intend to prove that the operator $\Psi$ has a fixed point. For all $n, m \in \mathbb{N}$, we may write

$$
\begin{aligned}
\| \Psi & \left(S_{n}(t)\right)-\Psi\left(S_{m}(t)\right) \| \\
= & \left\|S_{n+1}(t)-S_{m+1}(t)\right\| \\
= & \| S_{n}(t)+\mathbb{S T}^{-1}\left[\frac{1-\varrho+\varrho s}{M(\varrho)}\right. \\
& \left.\times \mathbb{S T}\left[\omega^{\varrho}-\delta^{\varrho} S_{n}(t) I_{n}(t)-\left(\rho^{\varrho}+v^{\varrho}\right) S_{n}(t)+\zeta^{\varrho} R_{n}(t)+\varpi^{\varrho} V_{n}(t)\right](s)\right] \\
& -S_{m}(t)-\mathbb{S T}^{-1}\left[\frac{1-\varrho+\varrho s}{M(\varrho)}\right. \\
& \left.\times \mathbb{S T}\left[\omega^{\varrho}-\delta^{\varrho} S_{m}(t) I_{m}(t)-\left(\rho^{\varrho}+v^{\varrho}\right) S_{m}(t)+\zeta^{\varrho} R_{m}(t)+\varpi^{\varrho} V_{m}(t)\right](s)\right] \| \\
\leq & \left\|S_{n}(t)-S_{m}(t)\right\|+\| \mathbb{S T}^{-1}\left[\frac { 1 - \varrho + \varrho s } { M ( \varrho ) } \mathbb { S T } \left[-\delta^{\varrho}\left(S_{n}(t) I_{n}(t)-S_{m}(t) I_{m}(t)\right)\right.\right. \\
& \left.\left.-\left(\rho^{\varrho}+v^{\varrho}\right)\left(S_{n}(t)-S_{m}(t)\right)+\zeta^{\varrho}\left(R_{n}(t)-R_{m}(t)\right)+\varpi^{\varrho}\left(V_{n}(t)-V_{m}(t)\right)\right](s)\right] \| \\
\leq & \left\|S_{n}(t)-S_{m}(t)\right\|+\mathbb{S T}^{-1}\left[\frac { 1 - \varrho + \varrho s } { M ( \varrho ) } \mathbb { S T } \left[\left\|-\delta^{\varrho} S_{n}(t)\left(I_{n}(t)-I_{m}(t)\right)\right\|\right.\right. \\
& +\left\|-\delta^{\varrho} I_{m}(t)\left(S_{n}(t)-S_{m}(t)\right)\right\| \\
& +\left\|-\left(\rho^{\varrho}+v^{\varrho}\right)\left(S_{n}(t)-S_{m}(t)\right)\right\|+\left\|\zeta^{\varrho}\left(R_{n}(t)-R_{m}(t)\right)\right\| \\
& \left.\left.+\left\|\varpi^{\varrho}\left(V_{n}(t)-V_{m}(t)\right)\right\|\right](s)\right] .
\end{aligned}
$$

Because of the same role of all four solutions, we will consider

$$
\left\|S_{n}(t)-S_{m}(t)\right\| \simeq\left\|I_{n}(t)-I_{m}(t)\right\| \simeq\left\|R_{n}(t)-R_{m}(t)\right\| \simeq\left\|V_{n}(t)-V_{m}(t)\right\| .
$$

Then from (43) and (44) we have

$$
\begin{aligned}
\left\|\Psi\left(S_{n}(t)\right)-\Psi\left(S_{m}(t)\right)\right\| \leq & \left\|S_{n}(t)-S_{m}(t)\right\| \\
& +\mathbb{S T}^{-1}\left[\frac { 1 - \varrho + \varrho s } { M ( \varrho ) } \mathbb { S T } \left[\left\|-\delta^{\varrho} S_{n}(t)\left(S_{n}(t)-S_{m}(t)\right)\right\|\right.\right. \\
& +\left\|-\delta^{\varrho} I_{m}(t)\left(S_{n}(t)-S_{m}(t)\right)\right\|+\left\|-\left(\rho^{\varrho}+v^{\varrho}\right)\left(S_{n}(t)-S_{m}(t)\right)\right\| \\
& \left.\left.+\left\|\zeta^{\varrho}\left(S_{n}(t)-S_{m}(t)\right)\right\|+\left\|\varpi^{\varrho}\left(S_{n}(t)-S_{m}(t)\right)\right\|\right](s)\right]
\end{aligned}
$$

Since $S_{n}, I_{m}, R_{n}$, and $V_{n}$ are convergent sequences, they are bounded. Hence there are constants $K_{1}^{*}, K_{2}^{*}, K_{3}^{*}$, and $K_{4}^{*}$ such that for all $t$ and $m, n \in \mathbb{N}$, we have

$$
\left\|S_{n}(t)\right\| \leq K_{1}^{*}, \quad\left\|I_{m}(t)\right\| \leq K_{2}^{*}, \quad\left\|R_{n}(t)\right\| \leq K_{3}^{*}, \quad\left\|V_{n}(t)\right\| \leq K_{4}^{*} .
$$


Therefore we obtain

$$
\begin{aligned}
\| \Psi & \left(S_{n}(t)\right)-\Psi\left(S_{m}(t)\right) \| \\
\leq & \left\|S_{n}(t)-S_{m}(t)\right\| \\
& +\mathbb{S T}^{-1}\left[\frac { 1 - \varrho + \varrho s } { M ( \varrho ) } \mathbb { S T } \left[-\delta^{\varrho} K_{1}^{*}\left\|S_{n}(t)-S_{m}(t)\right\|\right.\right. \\
& -\delta^{\varrho} K_{2}^{*}\left\|S_{n}(t)-S_{m}(t)\right\|-\left(\rho^{\varrho}+v^{\varrho}\right)\left\|S_{n}(t)-S_{m}(t)\right\| \\
& \left.\left.+\zeta^{\varrho}\left\|S_{n}(t)-S_{m}(t)\right\|+\varpi^{\varrho}\left\|S_{n}(t)-S_{m}(t)\right\|\right](s)\right] \\
= & \left(1-\delta^{\varrho} K_{1}^{*} \Phi_{1}(t)-\delta^{\varrho} K_{2}^{*} \Phi_{2}(t)-\left(\rho^{\varrho}+v^{\varrho}\right) \Phi_{3}(t)+\zeta^{\varrho} \Phi_{4}(t)+\varpi^{\varrho} \Phi_{5}(t)\right) \\
& \times\left\|S_{n}(t)-S_{m}(t)\right\|,
\end{aligned}
$$

where $\Phi_{j}, j=1,2, \ldots, 5$, are functions arising from $\mathbb{S T}^{-1}\left[\frac{1-\varrho+\varrho s}{M(\varrho)} \mathbb{S} \mathbb{T}[\cdot]\right]$. In the same manner, we get

$$
\begin{aligned}
\left\|\Psi\left(I_{n}(t)\right)-\Psi\left(I_{m}(t)\right)\right\| \leq & \left(1+\delta^{\varrho} K_{1}^{*} \Phi_{6}(t)+\delta^{\varrho} K_{2}^{*} \Phi_{7}(t)-\left(\rho^{\varrho}+\tau^{\varrho}+\kappa^{\varrho}\right) \Phi_{8}(t)\right) \\
& \times\left\|I_{n}(t)-I_{m}(t)\right\|, \\
\left\|\Psi\left(R_{n}(t)\right)-\Psi\left(R_{m}(t)\right)\right\| & \leq\left(1+\kappa^{\varrho} \Phi_{9}(t)-\left(\rho^{\varrho}+\zeta^{\varrho}\right) \Phi_{10}(t)\right)\left\|R_{n}(t)-R_{m}(t)\right\|,
\end{aligned}
$$

and

$$
\left\|\Psi\left(V_{n}(t)\right)-\Psi\left(V_{m}(t)\right)\right\| \leq\left(1+v^{\varrho} \Phi_{11}(t)-\left(\rho^{\varrho}+\varpi^{\varrho}\right) \Phi_{12}(t)\right)\left\|V_{n}(t)-V_{m}(t)\right\| .
$$

Under hypotheses (42), the self-map $\Psi$ is a contraction, and thus it possesses a fixed point. Now we claim that $\Psi$ satisfies all assumptions of Theorem 2.2. To prove this claim, we can easily assume that $K=(0,0,0)$ and

$$
k=\left\{\begin{array}{l}
1-\delta^{\varrho} K_{1}^{*} \Phi_{1}(t)-\delta^{\varrho} K_{2}^{*} \Phi_{2}(t)-\left(\rho^{\varrho}+v^{\varrho}\right) \Phi_{3}(t)+\zeta^{\varrho} \Phi_{4}(t)+\varpi^{\varrho} \Phi_{5}(t) \\
1+\delta^{\varrho} K_{1}^{*} \Phi_{6}(t)+\delta^{\varrho} K_{2}^{*} \Phi_{7}(t)-\left(\rho^{\varrho}+\tau^{\varrho}+\kappa^{\varrho}\right) \Phi_{8}(t) \\
1+\kappa^{\varrho} \Phi_{9}(t)-\left(\rho^{\varrho}+\zeta^{\varrho}\right) \Phi_{10}(t) \\
1+v^{\varrho} \Phi_{11}(t)-\left(\rho^{\varrho}+\varpi^{\varrho}\right) \Phi_{12}(t)
\end{array}\right.
$$

Then all assumptions of Theorem 2.2 are fulfilled, and so $\Psi$ is Picard $\Psi$-stable, and the proof is completed.

\section{Analytical solutions of model (8) by HATM method}

In this section, we implement the homotopy analysis transform method (HATM) to solve the fractional anthrax disease model (8). This method is an elegant combination of the standard Laplace transform method [38] and homotopy analysis method [39]. The advantage of this well-developed method is its flexible capability of combining two powerful methods to obtain exact and approximate analytical solutions for the existing fractional nonlinear equations. To solve the $\mathcal{C F}$-fractional anthrax disease model (8) by means of 
HATM, we first take the Laplace transform of both sides of fractional differential equations of $\mathcal{C F}$-system (8). Thus we have

$$
\left\{\begin{array}{l}
\mathbb{L}\left[{ }^{\mathcal{C F}} \mathcal{D}_{0}^{\varrho} S(t)\right](s)=\mathbb{L}\left[\omega^{\varrho}-\delta^{\varrho} S(t) I(t)-\left(\rho^{\varrho}+v^{\varrho}\right) S(t)+\zeta^{\varrho} R(t)+\varpi^{\varrho} V(t)\right](s), \\
\mathbb{L}\left[{ }^{\mathcal{C}} \mathcal{D}_{0}^{\varrho} I(t)\right](s)=\mathbb{L}\left[\delta^{\varrho} S(t) I(t)-\left(\rho^{\varrho}+\tau^{\varrho}+\kappa^{\varrho}\right) I(t)\right](s), \\
\mathbb{L}\left[{ }^{\mathcal{C}} \mathcal{D}_{0}^{\varrho} R(t)\right](s)=\mathbb{L}\left[\kappa^{\varrho} I(t)-\left(\rho^{\varrho}+\zeta^{\varrho}\right) R(t)\right](s), \\
\mathbb{L}\left[{ }^{\mathcal{C F}} \mathcal{D}_{0}^{\varrho} V(t)\right](s)=\mathbb{L}\left[v^{\varrho} S(t)-\left(\rho^{\varrho}+\varpi^{\varrho}\right) V(t)\right](s) .
\end{array}\right.
$$

Now by the definition of the Laplace transform of the fractional $\mathcal{C F}$-derivative we obtain

$$
\left\{\begin{array}{l}
\frac{s \mathbb{L}[S(t)]-S(0)}{s+\varrho(1-s)}=\mathbb{L}\left[\omega^{\varrho}-\delta^{\varrho} S(t) I(t)-\left(\rho^{\varrho}+v^{\varrho}\right) S(t)+\zeta^{\varrho} R(t)+\varpi^{\varrho} V(t)\right](s), \\
\frac{s \mathbb{L}[I(t)]-I(0)}{s+(1-s)}=\mathbb{L}\left[\delta^{\varrho} S(t) I(t)-\left(\rho^{\varrho}+\tau^{\varrho}+\kappa^{\varrho}\right) I(t)\right](s), \\
\frac{s \mathbb{L}[R(t)]-R(0)}{s+\varrho(1-s)}=\mathbb{L}\left[\kappa^{\varrho} I(t)-\left(\rho^{\varrho}+\zeta^{\varrho}\right) R(t)\right](s), \\
\frac{s \mathbb{L}[V(t)]-V(0)}{s+\varrho(1-s)}=\mathbb{L}\left[v^{\varrho} S(t)-\left(\rho^{\varrho}+\varpi^{\varrho}\right) V(t)\right](s) .
\end{array}\right.
$$

Rewriting these equalities, we get

$$
\left\{\begin{array}{l}
\mathbb{L}[S(t)]-\frac{\breve{S}_{0}}{s}-\frac{s+\varrho(1-s)}{s} \mathbb{L}\left[\omega^{\varrho}-\delta^{\varrho} S(t) I(t)-\left(\rho^{\varrho}+v^{\varrho}\right) S(t)\right. \\
\left.\quad+\zeta^{\varrho} R(t)+\varpi^{\varrho} V(t)\right](s)=0, \\
\mathbb{L}[I(t)]-\frac{\breve{I}_{0}}{s}-\frac{s+\varrho(1-s)}{s} \mathbb{L}\left[\delta^{\varrho} S(t) I(t)-\left(\rho^{\varrho}+\tau^{\varrho}+\kappa^{\varrho}\right) I(t)\right](s)=0, \\
\mathbb{L}[R(t)]-\frac{\breve{R}_{0}}{s}-\frac{s+\varrho(1-s)}{s} \mathbb{L}\left[\kappa^{\varrho} I(t)-\left(\rho^{\varrho}+\zeta^{\varrho}\right) R(t)\right](s)=0, \\
\mathbb{L}[V(t)]-\frac{\breve{V}_{0}}{s}-\frac{s+\varrho(1-s)}{s} \mathbb{L}\left[v^{\varrho} S(t)-\left(\rho^{\varrho}+\varpi^{\varrho}\right) V(t)\right](s)=0 .
\end{array}\right.
$$

By utilizing the homotopy analysis method we further find series solutions for the $\mathcal{C F}$ fractional anthrax disease model (8). To reach this goal, we consider $q \in[0,1]$ as the embedding parameter. The HAM technique is based on continuous mappings

$$
\mathcal{Q}_{1}(t ; q) \rightarrow S(t), \quad \mathcal{Q}_{2}(t ; q) \rightarrow I(t), \quad \mathcal{Q}_{3}(t ; q) \rightarrow R(t), \quad \mathcal{Q}_{4}(t ; q) \rightarrow V(t),
$$

so that as $q$ increases from 0 to $1, \mathcal{Q}_{j}(t ; q)(j=1,2,3,4)$ vary from the initial approximation to the exact solution. To observe this subject, we define the following nonlinear operators:

$$
\begin{aligned}
& \mathcal{K}_{1}\left(\mathcal{Q}_{1}(t ; q), \mathcal{Q}_{2}(t ; q), \mathcal{Q}_{3}(t ; q), \mathcal{Q}_{4}(t ; q)\right) \\
&:= \mathbb{L}\left[\mathcal{Q}_{1}(t ; q)\right]-\frac{\breve{S}_{0}}{s}-\frac{s+\varrho(1-s)}{s} \\
& \quad \times \mathbb{L}\left[\omega^{\varrho}-\delta^{\varrho} \mathcal{Q}_{1}(t ; q) \mathcal{Q}_{2}(t ; q)-\left(\rho^{\varrho}+v^{\varrho}\right) \mathcal{Q}_{1}(t ; q)+\zeta^{\varrho} \mathcal{Q}_{3}(t ; q)+\varpi^{\varrho} \mathcal{Q}_{4}(t ; q)\right](s) \\
& \mathcal{K}_{2}\left(\mathcal{Q}_{1}(t ; q), \mathcal{Q}_{2}(t ; q), \mathcal{Q}_{3}(t ; q), \mathcal{Q}_{4}(t ; q)\right) \\
&:=\mathbb{L}\left[\mathcal{Q}_{2}(t ; q)\right]-\frac{\breve{I}_{0}}{s}-\frac{s+\varrho(1-s)}{s} \\
& \quad \times \mathbb{L}\left[\delta^{\varrho} \mathcal{Q}_{1}(t ; q) \mathcal{Q}_{2}(t ; q)-\left(\rho^{\varrho}+\tau^{\varrho}+\kappa^{\varrho}\right) \mathcal{Q}_{2}(t ; q)\right](s) \\
& \mathcal{K}_{3}\left(\mathcal{Q}_{1}(t ; q), \mathcal{Q}_{2}(t ; q), \mathcal{Q}_{3}(t ; q), \mathcal{Q}_{4}(t ; q)\right) \\
&:= \mathbb{L}\left[\mathcal{Q}_{3}(t ; q)\right]-\frac{\breve{R}_{0}}{s}-\frac{s+\varrho(1-s)}{s}
\end{aligned}
$$




$$
\begin{aligned}
& \times \mathbb{L}\left[\kappa^{\varrho} \mathcal{Q}_{2}(t ; q)-\left(\rho^{\varrho}+\zeta^{\varrho}\right) \mathcal{Q}_{3}(t ; q)\right](s), \\
& \mathcal{K}_{4}\left(\mathcal{Q}_{1}(t ; q), \mathcal{Q}_{2}(t ; q), \mathcal{Q}_{3}(t ; q), \mathcal{Q}_{4}(t ; q)\right) \\
&:= \mathbb{L}\left[\mathcal{Q}_{4}(t ; q)\right]-\frac{\breve{V}_{0}}{s}-\frac{s+\varrho(1-s)}{s} \\
& \quad \times \mathbb{L}\left[v^{\varrho} \mathcal{Q}_{1}(t ; q)-\left(\rho^{\varrho}+\varpi^{\varrho}\right) \mathcal{Q}_{4}(t ; q)\right](s) .
\end{aligned}
$$

Then we construct the following collection of zero-order deformation equations [39]:

$$
\left\{\begin{array}{l}
(1-q) \mathbb{L}\left[\mathcal{Q}_{1}(t ; q)-\breve{S}_{0}\right]=q h H(t) \mathcal{K}_{1}\left(\mathcal{Q}_{1}(t ; q), \mathcal{Q}_{2}(t ; q), \mathcal{Q}_{3}(t ; q), \mathcal{Q}_{4}(t ; q)\right), \\
(1-q) \mathbb{L}\left[\mathcal{Q}_{2}(t ; q)-\breve{I}_{0}\right]=q h H(t) \mathcal{K}_{1}\left(\mathcal{Q}_{1}(t ; q), \mathcal{Q}_{2}(t ; q), \mathcal{Q}_{3}(t ; q), \mathcal{Q}_{4}(t ; q)\right), \\
(1-q) \mathbb{L}\left[\mathcal{Q}_{3}(t ; q)-\breve{R}_{0}\right]=q h H(t) \mathcal{K}_{1}\left(\mathcal{Q}_{1}(t ; q), \mathcal{Q}_{2}(t ; q), \mathcal{Q}_{3}(t ; q), \mathcal{Q}_{4}(t ; q)\right), \\
(1-q) \mathbb{L}\left[\mathcal{Q}_{4}(t ; q)-\breve{V}_{0}\right]=q h H(t) \mathcal{K}_{1}\left(\mathcal{Q}_{1}(t ; q), \mathcal{Q}_{2}(t ; q), \mathcal{Q}_{3}(t ; q), \mathcal{Q}_{4}(t ; q)\right)
\end{array}\right.
$$

supplemented with initial conditions

$$
\mathcal{Q}_{1}(0 ; q)=\breve{S}_{0}, \quad \mathcal{Q}_{2}(0 ; q)=\breve{I}_{0}, \quad \mathcal{Q}_{3}(0 ; q)=\breve{R}_{0}, \quad \mathcal{Q}_{4}(0 ; q)=\breve{V}_{0}
$$

where $q \in[0,1]$ is the embedding parameter, $h$ is a nonzero auxiliary parameter, $H$ is an auxiliary nonzero function, $\breve{S}_{0}, \breve{I}_{0}, \breve{R}_{0}$, and $\breve{V}_{0}$ are initial guesses of $S(t), I(t), R(t)$, and $V(t)$, $\mathcal{Q}_{j}(t ; q)(j=1,2,3,4)$ are unknown functions, and $\mathbb{L}$ is the Laplace linear operator. It is necessary to have great freedom to choose auxiliary things in HAM. It is obvious that by letting $q=0$ and $q=1$ we have

$$
\begin{cases}\mathcal{Q}_{1}(t ; 0)=\breve{S}_{0}, & \mathcal{Q}_{1}(t ; 1)=S(t) \\ \mathcal{Q}_{2}(t ; 0)=\breve{I}_{0}, & \mathcal{Q}_{2}(t ; 1)=I(t) \\ \mathcal{Q}_{3}(t ; 0)=\breve{R}_{0}, & \mathcal{Q}_{3}(t ; 1)=R(t) \\ \mathcal{Q}_{4}(t ; 0)=\breve{V}_{0}, & \mathcal{Q}_{4}(t ; 1)=V(t)\end{cases}
$$

Then we can observe that by increasing $q$ from 0 to 1 the solutions $\mathcal{Q}_{1}(t ; q), \mathcal{Q}_{2}(t ; q)$, $\mathcal{Q}_{3}(t ; q)$, and $\mathcal{Q}_{4}(t ; q)$ vary from the initial guesses $\breve{S}_{0}, \breve{I}_{0}, \breve{R}_{0}$, and $\breve{V}_{0}$ to $S(t), I(t), R(t)$, and $V(t)$, respectively. In this step, we expand the functions $\mathcal{Q}_{1}(t ; q), \mathcal{Q}_{2}(t ; q), \mathcal{Q}_{3}(t ; q)$, and $\mathcal{Q}_{4}(t ; q)$ by using Taylor's series with respect to $q$. Then we get

$$
\left\{\begin{array}{l}
\mathcal{Q}_{1}(t ; q)=\breve{S}_{0}+\sum_{r=1}^{\infty} S_{r}(t) q^{r} \\
\mathcal{Q}_{2}(t ; q)=\breve{I}_{0}+\sum_{r=1}^{\infty} I_{r}(t) q^{r} \\
\mathcal{Q}_{3}(t ; q)=\breve{R}_{0}+\sum_{r=1}^{\infty} R_{r}(t) q^{r} \\
\mathcal{Q}_{4}(t ; q)=\breve{V}_{0}+\sum_{r=1}^{\infty} V_{r}(t) q^{r}
\end{array}\right.
$$

where $S_{r}(t)=\left.\frac{1}{r !} \frac{\partial^{r} \mathcal{Q}_{1}(t ; q)}{\partial q^{r}}\right|_{q=0}, I_{r}(t)=\left.\frac{1}{r !} \frac{\partial^{r} \mathcal{Q}_{2}(t ; q)}{\partial q^{r}}\right|_{q=0}, R_{r}(t)=\left.\frac{1}{r !} \frac{\partial^{r} \mathcal{Q}_{3}(t ; q)}{\partial q^{r}}\right|_{q=0}$, and $V_{r}(t)=\frac{1}{r !} \times$ $\left.\frac{\partial^{r} \mathcal{Q}_{4}(t ; q)}{\partial q^{r}}\right|_{q=0}$ are the constant coefficients of the series (48). If we choose a suitable auxiliary linear operator, suitable initial guesses, a suitable auxiliary parameter $h$, and a suitable auxiliary function $H$, then the series (48) is convergent at $q=1$, as proved by Liao [39] (also 
see $[52,53])$. Thus we have

$$
\left\{\begin{array} { l } 
{ \mathcal { Q } _ { 1 } ( t ; q ) = \breve { S } _ { 0 } + \sum _ { r = 1 } ^ { \infty } S _ { r } ( t ) , } \\
{ \mathcal { Q } _ { 2 } ( t ; q ) = \breve { I } _ { 0 } + \sum _ { r = 1 } ^ { \infty } I _ { r } ( t ) , } \\
{ \mathcal { Q } _ { 3 } ( t ; q ) = \breve { R } _ { 0 } + \sum _ { r = 1 } ^ { \infty } R _ { r } ( t ) , } \\
{ \mathcal { Q } _ { 4 } ( t ; q ) = \breve { V } _ { 0 } + \sum _ { r = 1 } ^ { \infty } V _ { r } ( t ) , }
\end{array} \Longrightarrow \left\{\begin{array}{l}
S(t)=\breve{S}_{0}+\sum_{r=1}^{\infty} S_{r}(t), \\
I(t)=\breve{I}_{0}+\sum_{r=1}^{\infty} I_{r}(t), \\
R(t)=\breve{R}_{0}+\sum_{r=1}^{\infty} R_{r}(t) \\
V(t)=\breve{V}_{0}+\sum_{r=1}^{\infty} V_{r}(t)
\end{array}\right.\right.
$$

which must be the solutions of the $\mathcal{C} \mathcal{F}$-fractional anthrax disease model (8). Now we produce the following $r$ th-order deformation equations. Define the vectors

$$
\begin{aligned}
& \vec{S}_{r}(t)=\left\{S_{0}(t), S_{1}(t), \ldots, S_{r}(t)\right\}(r=1,2,3, \ldots), \\
& \vec{I}_{r}(t)=\left\{I_{0}(t), I_{1}(t), \ldots, I_{r}(t)\right\} \quad(r=1,2,3, \ldots), \\
& \vec{R}_{r}(t)=\left\{R_{0}(t), R_{1}(t), \ldots, R_{r}(t)\right\} \quad(r=1,2,3, \ldots), \\
& \vec{V}_{r}(t)=\left\{V_{0}(t), V_{1}(t), \ldots, V_{r}(t)\right\} \quad(r=1,2,3, \ldots) .
\end{aligned}
$$

We differentiate the zero-order deformation equations (47) $r$ times with respect to the embedding parameter $q$. Next, we take $q=0$ and finally divide them by $r$.. In this case, we obtain the following $r$ th-order linear deformation equations:

$$
\left\{\begin{array}{l}
\mathbb{L}\left[S_{r}(t)-\sigma_{r} S_{r-1}(t)\right]=h H \Re_{S, r}\left(\vec{S}_{r-1}(t), \vec{I}_{r-1}(t), \vec{R}_{r-1}(t), \vec{V}_{r-1}(t)\right) \quad(r=1,2,3, \ldots), \\
\mathbb{L}\left[I_{r}(t)-\sigma_{r} I_{r-1}(t)\right]=h H \Re_{I, r}\left(\vec{S}_{r-1}(t), \vec{I}_{r-1}(t), \vec{R}_{r-1}(t), \vec{V}_{r-1}(t)\right) \quad(r=1,2,3, \ldots), \\
\mathbb{L}\left[R_{r}(t)-\sigma_{r} R_{r-1}(t)\right]=h H \Re_{R, r}\left(\vec{S}_{r-1}(t), \vec{I}_{r-1}(t), \vec{R}_{r-1}(t), \vec{V}_{r-1}(t)\right) \quad(r=1,2,3, \ldots), \\
\mathbb{L}\left[V_{r}(t)-\sigma_{r} V_{r-1}(t)\right]=h H \Re_{V, r}\left(\vec{S}_{r-1}(t), \vec{I}_{r-1}(t), \vec{R}_{r-1}(t), \vec{V}_{r-1}(t)\right) \quad(r=1,2,3, \ldots),
\end{array}\right.
$$

furnished with initial values

$$
S_{0}(t)=\breve{S}_{0}, \quad I_{0}(t)=\breve{I}_{0}, \quad R_{0}(t)=\breve{R}_{0}, \quad V_{0}(t)=\breve{V}_{0},
$$

where

$$
\sigma_{r}= \begin{cases}0, & r \leq 1, \\ 1, & r>1,\end{cases}
$$

and

$$
\begin{aligned}
\Re_{S, r} & \left(\vec{S}_{r-1}(t), \vec{I}_{r-1}(t), \vec{R}_{r-1}(t), \vec{V}_{r-1}(t)\right) \\
= & \mathbb{L}\left[S_{r-1}(t)\right]-\frac{\breve{S}_{0}}{s}\left(1-\sigma_{r}\right)-\frac{s+\varrho(1-s)}{s} \\
& \times \mathbb{L}\left[\omega^{\varrho}-\delta^{\varrho} S_{r-1}(t) I_{r-1}(t)-\left(\rho^{\varrho}+v^{\varrho}\right) S_{r-1}(t)\right. \\
& \left.+\zeta^{\varrho} R_{r-1}(t)+\varpi^{\varrho} V_{r-1}(t)\right](s), \\
\mathfrak{R}_{I, r}\left(\vec{S}_{r-1}(t), \vec{I}_{r-1}(t), \vec{R}_{r-1}(t), \vec{V}_{r-1}(t)\right) & \\
= & \mathbb{L}\left[I_{r-1}(t)\right]-\frac{\breve{I}_{0}}{s}\left(1-\sigma_{r}\right)-\frac{s+\varrho(1-s)}{s}
\end{aligned}
$$




$$
\begin{aligned}
& \times \mathbb{L}\left[\delta^{\varrho} S_{r-1}(t) I_{r-1}(t)-\left(\rho^{\varrho}+\tau^{\varrho}+\kappa^{\varrho}\right) I_{r-1}(t)\right](s), \\
\mathfrak{R}_{R, r} & \left(\vec{S}_{r-1}(t), \vec{I}_{r-1}(t), \vec{R}_{r-1}(t), \vec{V}_{r-1}(t)\right) \\
= & \mathbb{L}\left[R_{r-1}(t)\right]-\frac{\breve{R}_{0}}{s}\left(1-\sigma_{r}\right)-\frac{s+\varrho(1-s)}{s} \\
& \times \mathbb{L}\left[\kappa^{\varrho} I_{r-1}(t)-\left(\rho^{\varrho}+\zeta^{\varrho}\right) R_{r-1}(t)\right](s),
\end{aligned}
$$

and

$$
\begin{aligned}
& \mathfrak{R}_{V, r}\left(\vec{S}_{r-1}(t), \vec{I}_{r-1}(t), \vec{R}_{r-1}(t), \vec{V}_{r-1}(t)\right) \\
&= \mathbb{L}\left[V_{r-1}(t)\right]-\frac{\breve{V}_{0}}{s}\left(1-\sigma_{r}\right)-\frac{s+\varrho(1-s)}{s} \\
& \quad \times \mathbb{L}\left[v^{\varrho} S_{r-1}(t)-\left(\rho^{\varrho}+\varpi^{\varrho}\right) V_{r-1}(t)\right](s) .
\end{aligned}
$$

Applying the inverse Laplace transform to both sides of (50), we obtain

$$
\begin{aligned}
& S_{r}(t)=\sigma_{r} S_{r-1}(t)+h H \mathbb{L}^{-1}\left[\Re_{S, r}\left(\vec{S}_{r-1}(t), \vec{I}_{r-1}(t), \vec{R}_{r-1}(t), \vec{V}_{r-1}(t)\right)\right], \\
& I_{r}(t)=\sigma_{r} I_{r-1}(t)+h H \mathbb{L}^{-1}\left[\Re_{I, r}\left(\vec{S}_{r-1}(t), \vec{I}_{r-1}(t), \vec{R}_{r-1}(t), \vec{V}_{r-1}(t)\right)\right], \\
& R_{r}(t)=\sigma_{r} R_{r-1}(t)+h H \mathbb{L}^{-1}\left[\Re_{R, r}\left(\vec{S}_{r-1}(t), \vec{I}_{r-1}(t), \vec{R}_{r-1}(t), \vec{V}_{r-1}(t)\right)\right], \\
& V_{r}(t)=\sigma_{r} V_{r-1}(t)+h H \mathbb{L}^{-1}\left[\Re_{V, r}\left(\vec{S}_{r-1}(t), \vec{I}_{r-1}(t), \vec{R}_{r-1}(t), \vec{V}_{r-1}(t)\right)\right]
\end{aligned}
$$

for $r=1,2,3, \ldots$. For convenience, we can consider the nonzero auxiliary function $H$ to be equal to unity. Now, if we solve equation (52) for $r=1$, then in view of initial conditions (51), we have

$$
\begin{aligned}
S_{1}(t)= & \sigma_{1} S_{0}(t)+h H \mathbb{L}^{-1}\left[\Re_{S, 1}\left(\vec{S}_{0}(t), \vec{I}_{0}(t), \vec{R}_{0}(t), \vec{V}_{0}(t)\right)\right] \\
= & h H \mathbb{L}^{-1}\left[\mathbb{L}\left[\breve{S}_{0}\right]-\frac{\breve{S}_{0}}{s}\left(1-\sigma_{1}\right)\right. \\
& \left.-\frac{s+\varrho(1-s)}{s} \mathbb{L}\left[\omega^{\varrho}-\delta^{\varrho} \breve{S}_{0} \breve{I}_{0}-\left(\rho^{\varrho}+v^{\varrho}\right) \breve{S}_{0}+\zeta^{\varrho} \breve{R}_{0}+\varpi^{\varrho} \breve{V}_{0}\right]\right] \\
= & -h H \mathbb{L}^{-1}\left[\frac{s+\varrho(1-s)}{s^{2}}\left(\omega^{\varrho}-\delta^{\varrho} \breve{S}_{0} \breve{I}_{0}-\left(\rho^{\varrho}+v^{\varrho}\right) \breve{S}_{0}+\zeta^{\varrho} \breve{R}_{0}+\varpi^{\varrho} \breve{V}_{0}\right)\right] \\
= & -\hat{\Delta}_{1} h H \mathbb{L}^{-1}\left[\frac{s+\varrho(1-s)}{s^{2}}\right] \\
= & -\hat{\Delta}_{1} h H(1+\varrho(t-1)),
\end{aligned}
$$

where $\hat{\Delta}_{1}=\omega^{\varrho}-\delta^{\varrho} \breve{S}_{0} \breve{I}_{0}-\left(\rho^{\varrho}+v^{\varrho}\right) \breve{S}_{0}+\zeta^{\varrho} \breve{R}_{0}+\varpi^{\varrho} \breve{V}_{0}$. Hence, continuing similar computations on equations (53)-(55), we get

$$
\left\{\begin{array}{l}
I_{1}(t)=-\hat{\Delta}_{2} h H(1+\varrho(t-1)), \\
R_{1}(t)=-\hat{\Delta}_{3} h H(1+\varrho(t-1)), \\
V_{1}(t)=-\hat{\Delta}_{4} h H(1+\varrho(t-1)),
\end{array}\right.
$$


where $\hat{\Delta}_{2}=\delta^{\varrho} \breve{S}_{0} \breve{I}_{0}-\left(\rho^{\varrho}+\tau^{\varrho}+\kappa^{\varrho}\right) \breve{I}_{0}, \hat{\Delta}_{3}=\kappa^{\varrho} \breve{I}_{0}-\left(\rho^{\varrho}+\zeta^{\varrho}\right) \breve{R}_{0}$, and $\hat{\Delta}_{4}=v^{\varrho} \breve{S}_{0}-\left(\rho^{\varrho}+\varpi^{\varrho}\right) \breve{V}_{0}$. Again, if we solve equation (52) for $r=2$, then in view of (56)-(57), we have

$$
\begin{aligned}
S_{2}(t)= & S_{1}(t)-\hat{\Delta}_{1} h^{2} H^{2}(1+\varrho(t-1))-h H \omega^{\varrho}(1+\varrho(t-1)) \\
& +\hat{\Delta}_{1} \hat{\Delta}_{2} h^{3} H^{3} \delta^{\varrho}(1+3 \varrho(t-1) \\
& \left.+\varrho^{2}\left(2 t^{2}-6 t+3\right)+\varrho^{3}\left(\frac{t^{3}}{3}-2 t^{2}+3 t-1\right)\right) \\
& -\hat{\Delta}_{1} h^{2} H^{2}\left(\rho^{\varrho}+v^{\varrho}\right)\left(1+2 \varrho(t-1)+\varrho^{2}\left(\frac{t^{2}}{2}-2 t+1\right)\right) \\
& +\hat{\Delta}_{3} h^{2} H^{2} \zeta^{\varrho}\left(1+2 \varrho(t-1)+\varrho^{2}\left(\frac{t^{2}}{2}-2 t+1\right)\right) \\
& +\hat{\Delta}_{4} h^{2} H^{2} \varpi^{\varrho}\left(1+2 \varrho(t-1)+\varrho^{2}\left(\frac{t^{2}}{2}-2 t+1\right)\right) .
\end{aligned}
$$

Similarly, by solving the equations (53)-(55) for $r=2$ we get the following functions with respect to $t$ :

$$
\begin{aligned}
I_{2}(t)= & I_{1}(t)-\hat{\Delta}_{2} h^{2} H^{2}(1+\varrho(t-1)) \\
& -\hat{\Delta}_{1} \hat{\Delta}_{2} h^{3} H^{3} \delta^{\varrho}(1+3 \varrho(t-1) \\
& \left.+\varrho^{2}\left(2 t^{2}-6 t+3\right)+\varrho^{3}\left(\frac{t^{3}}{3}-2 t^{2}+3 t-1\right)\right) \\
& -\hat{\Delta}_{1} h^{2} H^{2}\left(\rho^{\varrho}+\tau^{\varrho}+\kappa^{\varrho}\right)\left(1+2 \varrho(t-1)+\varrho^{2}\left(\frac{t^{2}}{2}-2 t+1\right)\right), \\
R_{2}(t)= & R_{1}(t)-\hat{\Delta}_{3} h^{2} H^{2}(1+\varrho(t-1)) \\
& +\hat{\Delta}_{2} h^{2} H^{2} \kappa^{\varrho}\left(1+2 \varrho(t-1)+\varrho^{2}\left(\frac{t^{2}}{2}-2 t+1\right)\right) \\
& -\hat{\Delta}_{3} h^{2} H^{2}\left(\rho^{\varrho}+\zeta^{\varrho}\right)\left(1+2 \varrho(t-1)+\varrho^{2}\left(\frac{t^{2}}{2}-2 t+1\right)\right),
\end{aligned}
$$

and

$$
\begin{aligned}
V_{2}(t)= & V_{1}(t)-\hat{\Delta}_{4} h^{2} H^{2}(1+\varrho(t-1)) \\
& +\hat{\Delta}_{1} h^{2} H^{2} v^{\varrho}\left(1+2 \varrho(t-1)+\varrho^{2}\left(\frac{t^{2}}{2}-2 t+1\right)\right) \\
& -\hat{\Delta}_{4} h^{2} H^{2}\left(\rho^{\varrho}+\varpi^{\varrho}\right)\left(1+2 \varrho(t-1)+\varrho^{2}\left(\frac{t^{2}}{2}-2 t+1\right)\right) .
\end{aligned}
$$

According to the series (49), if we continue this precess and solve equations (52)-(55) for $r=3,4, \ldots$, then by (56)-(61) the series solutions for the $\mathcal{C F}$-fractional anthrax disease 
model (8) are given by

$$
\begin{aligned}
& S(t)=\breve{S}_{0}+\sum_{r=1}^{\infty} S_{r}(t)=\breve{S}_{0}+S_{1}(t)+S_{2}(t)+\cdots=\breve{S}_{0}-\hat{\Delta}_{1} h H(1+\varrho(t-1))+\cdots, \\
& I(t)=\breve{I}_{0}+\sum_{r=1}^{\infty} I_{r}(t)=\breve{I}_{0}+I_{1}(t)+I_{2}(t)+\cdots=\breve{I}_{0}-\hat{\Delta}_{2} h H(1+\varrho(t-1))+\cdots, \\
& R(t)=\breve{R}_{0}+\sum_{r=1}^{\infty} R_{r}(t)=\breve{R}_{0}+R_{1}(t)+R_{2}(t)+\cdots=\breve{R}_{0}-\hat{\Delta}_{3} h H(1+\varrho(t-1))+\cdots, \\
& V(t)=\breve{V}_{0}+\sum_{r=1}^{\infty} V_{r}(t)=\breve{V}_{0}+V_{1}(t)+V_{2}(t)+\cdots=\breve{V}_{0}-\hat{\Delta}_{4} h H(1+\varrho(t-1))+\cdots,
\end{aligned}
$$

where the constants $\hat{\Delta}_{1}, \hat{\Delta}_{2}, \hat{\Delta}_{3}$, and $\hat{\Delta}_{4}$ were introduced above.

\section{Convergence analysis of HATM for the $\mathcal{C} \mathcal{F}$-model}

In this section, we prove the convergence of HATM method utilized for the fractional $\mathcal{C F}$-system (46) of the anthrax disease model.

Theorem 8.1 Let $\sum_{r=0}^{\infty} S_{r}(t), \sum_{r=0}^{\infty} I_{r}(t), \sum_{r=0}^{\infty} R_{r}(t)$, and $\sum_{r=0}^{\infty} V_{r}(t)$ be uniformly convergent series approaching to $S(t), I(t), R(t)$, and $V(t)$, respectively, where $S_{r}(t), I_{r}(t), R_{r}(t)$, and $V_{r}(t)$ belonging to $\mathcal{L}\left(\mathbb{R}^{+}\right)$are produced by the rth-order deformation equations (50), and, in addition, $\sum_{r=0}^{\infty} \mathcal{C F} \mathcal{D}_{0}^{\varrho} S_{r}(t), \sum_{r=0}^{\infty}{ }^{\mathcal{C F}} \mathcal{D}_{0}^{\varrho} I_{r}(t), \sum_{r=0}^{\infty} \mathcal{C F} \mathcal{D}_{0}^{\varrho} R_{r}(t)$, and $\sum_{r=0}^{\infty}{ }^{\mathcal{C F}} \mathcal{D}_{0}^{\varrho} V_{r}(t)$ also are convergent series. Then the functions $S(t), I(t), R(t)$, and $V(t)$ are exact solutions of the fractional $\mathcal{C} \mathcal{F}_{\text {-system }}(46)$ of the anthrax disease model.

Proof Suppose that $\sum_{r=0}^{\infty} S_{r}(t)$ is an uniformly convergent series approaching to $S(t)$. Then, it is evident that $\lim _{r \rightarrow \infty} S_{r}(t)=0$ for any $t \in \mathbb{R}^{+}$. Since the Laplace operator is linear, we have

$$
\begin{array}{rl}
\sum_{r=1}^{n} & \mathbb{L}\left[S_{r}(t)-\sigma_{r} S_{r-1}(t)\right] \\
& =\sum_{r=1}^{n}\left(\mathbb{L}\left[S_{r}(t)\right]-\sigma_{r} \mathbb{L}\left[S_{r-1}(t)\right]\right) \\
& =\mathbb{L}\left[S_{1}(t)\right]+\left(\mathbb{L}\left[S_{2}(t)\right]-\mathbb{L}\left[S_{1}(t)\right]\right)+\cdots+\left(\mathbb{L}\left[S_{n}(t)\right]-\mathbb{L}\left[S_{n-1}(t)\right]\right) \\
& =\mathbb{L}\left[S_{n}(t)\right] .
\end{array}
$$

Therefore we get

$$
\sum_{r=1}^{\infty} \mathbb{L}\left[S_{r}(t)-\sigma_{r} S_{r-1}(t)\right]=\lim _{n \rightarrow \infty} \mathbb{L}\left[S_{n}(t)\right]=\mathbb{L}\left[\lim _{n \rightarrow \infty} S_{n}(t)\right]=0,
$$

and so

$$
\sum_{r=1}^{\infty} \mathbb{L}\left[S_{r}(t)-\sigma_{r} S_{r-1}(t)\right]=h H \sum_{r=1}^{\infty} \Re_{S, r}\left(\vec{S}_{r-1}(t), \vec{I}_{r-1}(t), \vec{R}_{r-1}(t), \vec{V}_{r-1}(t)\right)=0
$$


Since $h \neq 0$ and $H \neq 0$, this yields

$$
\sum_{r=1}^{\infty} \Re_{S, r}\left(\vec{S}_{r-1}(t), \vec{I}_{r-1}(t), \vec{R}_{r-1}(t), \vec{V}_{r-1}(t)\right)=0
$$

Similarly, we can prove that

$$
\begin{aligned}
& \sum_{r=1}^{\infty} \mathfrak{R}_{I, r}\left(\vec{S}_{r-1}(t), \vec{I}_{r-1}(t), \vec{R}_{r-1}(t), \vec{V}_{r-1}(t)\right)=0, \\
& \sum_{r=1}^{\infty} \Re_{R, r}\left(\vec{S}_{r-1}(t), \vec{I}_{r-1}(t), \vec{R}_{r-1}(t), \vec{V}_{r-1}(t)\right)=0, \\
& \sum_{r=1}^{\infty} \Re_{V, r}\left(\vec{S}_{r-1}(t), \vec{I}_{r-1}(t), \vec{R}_{r-1}(t), \vec{V}_{r-1}(t)\right)=0 .
\end{aligned}
$$

Now, from Equation (63) we have

$$
\begin{aligned}
0= & \sum_{r=1}^{\infty} \mathfrak{R}_{S, r}\left(\vec{S}_{r-1}(t), \vec{I}_{r-1}(t), \vec{R}_{r-1}(t), \vec{V}_{r-1}(t)\right) \\
= & \sum_{r=1}^{\infty}\left(\mathbb{L}\left[S_{r-1}(t)\right]-\frac{\breve{S}_{0}}{s}\left(1-\sigma_{r}\right)-\frac{s+\varrho(1-s)}{s}\right. \\
& \times \mathbb{L}\left[\omega^{\varrho}-\delta^{\varrho} S_{r-1}(t) I_{r-1}(t)-\left(\rho^{\varrho}+v^{\varrho}\right) S_{r-1}(t)\right. \\
& \left.\left.+\zeta^{\varrho} R_{r-1}(t)+\varpi^{\varrho} V_{r-1}(t)\right](s)\right) \\
= & {\left[\sum_{r=1}^{\infty} S_{r-1}(t)\right]-\frac{\breve{S}_{0}}{s} \sum_{r=1}^{\infty}\left(1-\sigma_{r}\right)-\frac{s+\varrho(1-s)}{s} } \\
& \times \mathbb{L}\left[\sum _ { r = 1 } ^ { \infty } \left(\omega^{\varrho}-\delta^{\varrho} S_{r-1}(t) I_{r-1}(t)\right.\right. \\
& \left.\left.-\left(\rho^{\varrho}+v^{\varrho}\right) S_{r-1}(t)+\zeta^{\varrho} R_{r-1}(t)+\varpi^{\varrho} V_{r-1}(t)\right)\right](s) \\
= & \mathbb{L}[S(t)]-\frac{\breve{S}_{0}}{s}-\frac{s+\varrho(1-s)}{s} \mathbb{L}\left[\omega^{\varrho}-\delta^{\varrho} S(t) I(t)\right. \\
& \left.-\left(\rho^{\varrho}+v^{\varrho}\right) S(t)+\zeta^{\varrho} R(t)+\varpi^{\varrho} V(t)\right](s) .
\end{aligned}
$$

Similarly, from (64)-(66) we have

$$
\begin{aligned}
0= & \sum_{r=1}^{\infty} \Re_{I, r}\left(\vec{S}_{r-1}(t), \vec{I}_{r-1}(t), \vec{R}_{r-1}(t), \vec{V}_{r-1}(t)\right) \\
= & \sum_{r=1}^{\infty}\left(\mathbb{L}\left[I_{r-1}(t)\right]-\frac{\breve{I}_{0}}{s}\left(1-\sigma_{r}\right)-\frac{s+\varrho(1-s)}{s}\right. \\
& \left.\times \mathbb{L}\left[\delta^{\varrho} S_{r-1}(t) I_{r-1}(t)-\left(\rho^{\varrho}+\tau^{\varrho}+\kappa^{\varrho}\right) I_{r-1}(t)\right](s)\right)
\end{aligned}
$$




$$
\begin{aligned}
= & \mathbb{L}\left[\sum_{r=1}^{\infty} I_{r-1}(t)\right]-\frac{\breve{I}_{0}}{s} \sum_{r=1}^{\infty}\left(1-\sigma_{r}\right)-\frac{s+\varrho(1-s)}{s} \\
& \times \mathbb{L}\left[\sum_{r=1}^{\infty}\left(\delta^{\varrho} S_{r-1}(t) I_{r-1}(t)-\left(\rho^{\varrho}+\tau^{\varrho}+\kappa^{\varrho}\right) I_{r-1}(t)\right)\right](s) \\
= & \mathbb{L}[I(t)]-\frac{\breve{I}_{0}}{s}-\frac{s+\varrho(1-s)}{s} \mathbb{L}\left[\delta^{\varrho} S(t) I(t)-\left(\rho^{\varrho}+\tau^{\varrho}+\kappa^{\varrho}\right) I(t)\right](s) \\
0= & \sum_{r=1}^{\infty} \Re_{R, r}\left(\vec{S}_{r-1}(t), \vec{I}_{r-1}(t), \vec{R}_{r-1}(t), \vec{V}_{r-1}(t)\right) \\
= & \sum_{r=1}^{\infty}\left(\mathbb{L}\left[R_{r-1}(t)\right]-\frac{\breve{R}_{0}}{s}\left(1-\sigma_{r}\right)-\frac{s+\varrho(1-s)}{s}\right. \\
& \left.\times \mathbb{L}\left[\kappa^{\varrho} I_{r-1}(t)-\left(\rho^{\varrho}+\zeta^{\varrho}\right) R_{r-1}(t)\right](s)\right) \\
= & \mathbb{L}\left[\sum_{r=1}^{\infty} R_{r-1}(t)\right]-\frac{\breve{R}_{0}}{s} \sum_{r=1}^{\infty}\left(1-\sigma_{r}\right)-\frac{s+\varrho(1-s)}{s} \\
& \times \mathbb{L}\left[\sum_{r=1}^{\infty}\left(\kappa^{\varrho} I_{r-1}(t)-\left(\rho^{\varrho}+\zeta^{\varrho}\right) R_{r-1}(t)\right)\right](s) \\
= & \mathbb{L}[R(t)]-\frac{\breve{R}_{0}}{s}-\frac{s+\varrho(1-s)}{s} \mathbb{L}\left[\kappa^{\varrho} I(t)-\left(\rho^{\varrho}+\zeta^{\varrho}\right) R(t)\right](s),
\end{aligned}
$$

and

$$
\begin{aligned}
0= & \sum_{r=1}^{\infty} \mathfrak{R}_{V, r}\left(\vec{S}_{r-1}(t), \vec{I}_{r-1}(t), \vec{R}_{r-1}(t), \vec{V}_{r-1}(t)\right) \\
= & \sum_{r=1}^{\infty}\left(\mathbb{L}\left[V_{r-1}(t)\right]-\frac{\breve{V}_{0}}{s}\left(1-\sigma_{r}\right)-\frac{s+\varrho(1-s)}{s}\right. \\
& \left.\times \mathbb{L}\left[v^{\varrho} S_{r-1}(t)-\left(\rho^{\varrho}+\varpi^{\varrho}\right) V_{r-1}(t)\right](s)\right) \\
= & \mathbb{L}\left[\sum_{r=1}^{\infty} V_{r-1}(t)\right]-\frac{\breve{V}_{0}}{s} \sum_{r=1}^{\infty}\left(1-\sigma_{r}\right)-\frac{s+\varrho(1-s)}{s} \\
& \times \mathbb{L}\left[\sum_{r=1}^{\infty}\left(v^{\varrho} S_{r-1}(t)-\left(\rho^{\varrho}+\varpi^{\varrho}\right) V_{r-1}(t)\right)\right](s) \\
= & \mathbb{L}[V(t)]-\frac{\breve{V}_{0}}{s}-\frac{s+\varrho(1-s)}{s} \mathbb{L}\left[v^{\varrho} S(t)-\left(\rho^{\varrho}+\varpi^{\varrho}\right) V(t)\right](s) .
\end{aligned}
$$

Therefore $S(t), I(t), R(t)$, and $V(t)$ are solutions of the fractional $\mathcal{C F}$-system (46) of the anthrax disease model. The proof is completed.

\section{Numerical simulations}

In this section, we present the results of numerical simulations based on the theoretical findings for the fractional anthrax disease $\mathcal{C F}$-system (8) over a period of $t=50$ months. In other words, we numerically calculate $S(t), I(t), R(t)$, and $V(t)$ at integer and noninteger orders $\varrho=1, \varrho=0.99, \varrho=0.97$, and $\varrho=0.95$. These solution functions for the fractional 


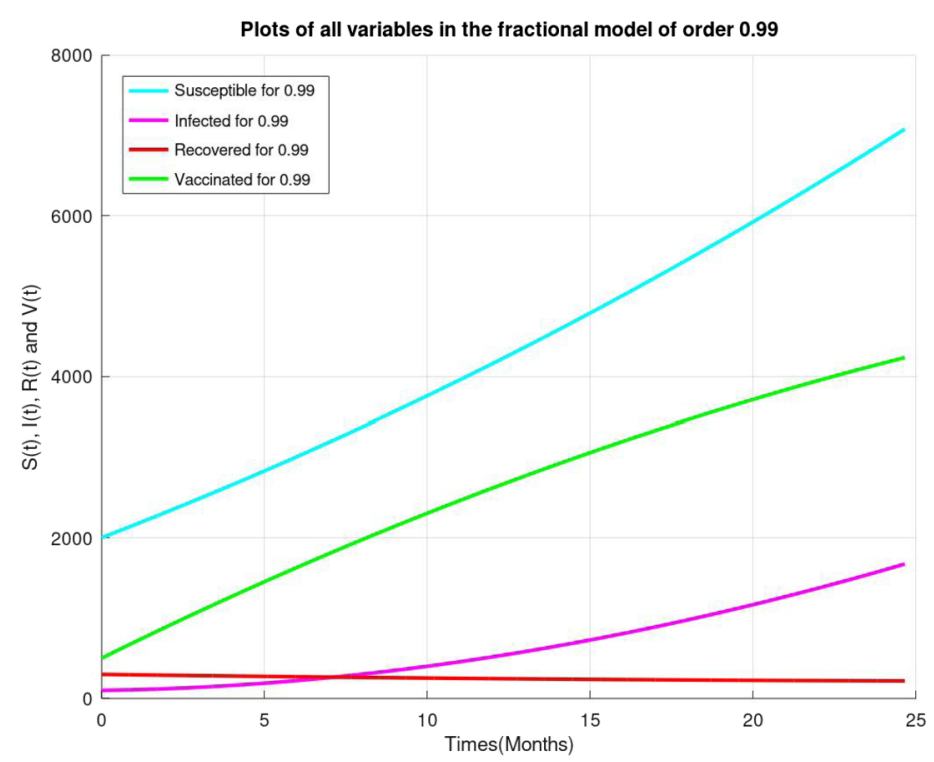

Figure 1 An illustration of the total number of each class of animal populations $S(t), /(t), R(t)$, and $V(t)$ for order $\varrho=0.99$

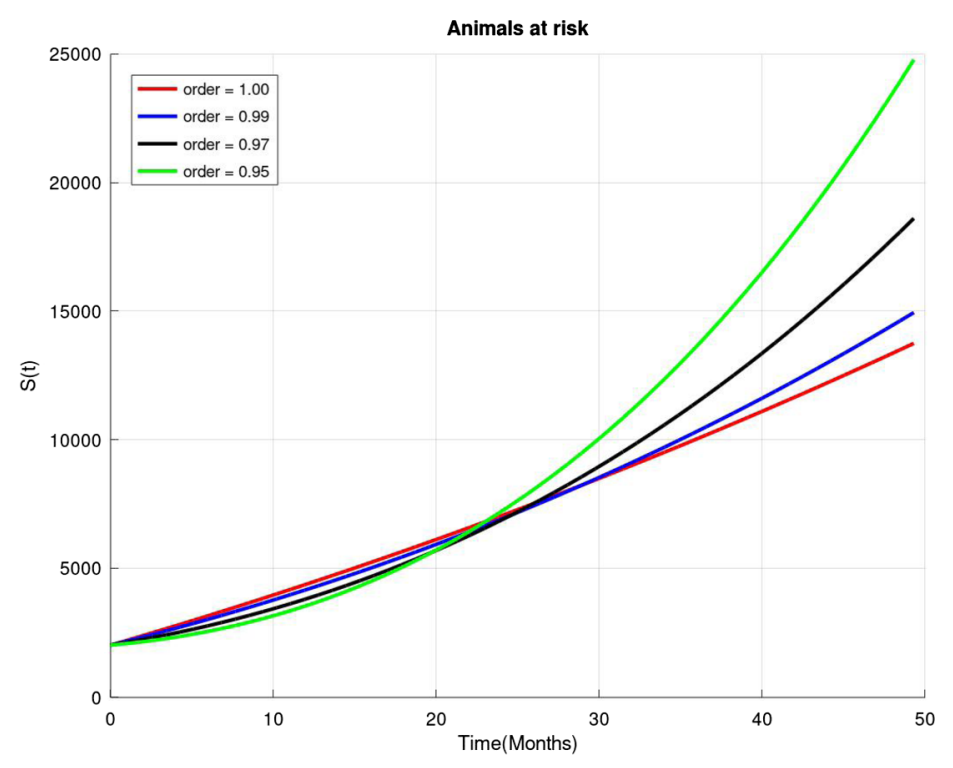

Figure 2 An illustration of the total number of susceptible animals $S(t)$ for different orders $\varrho=1, \varrho=0.99$, $\varrho=0.97$, and $\varrho=0.95$ during 50 months

anthrax disease $\mathcal{C} \mathcal{F}$-model (8) are obtained by implementing HATM technique. We use distinct values of nonnegative parameters $\omega=200, \delta=0.0001, \rho=0.001, v=0.1, \zeta=0.02$, $\varpi=0.003$, and $\kappa=0.01$ and select the initial values $\breve{S}_{0}=2000, \breve{I}_{0}=100, \breve{R}_{0}=300$, and $\breve{V}_{0}=500$. Note that these numerical values for parameters are taken from the existing data given in $[29,33,35,36]$.

Figure 1 shows the total number of each class of animal populations $S(t), I(t), R(t)$, and $V(t)$ during a time interval including 25 months for the fractional order $\varrho=0.99$. Based 


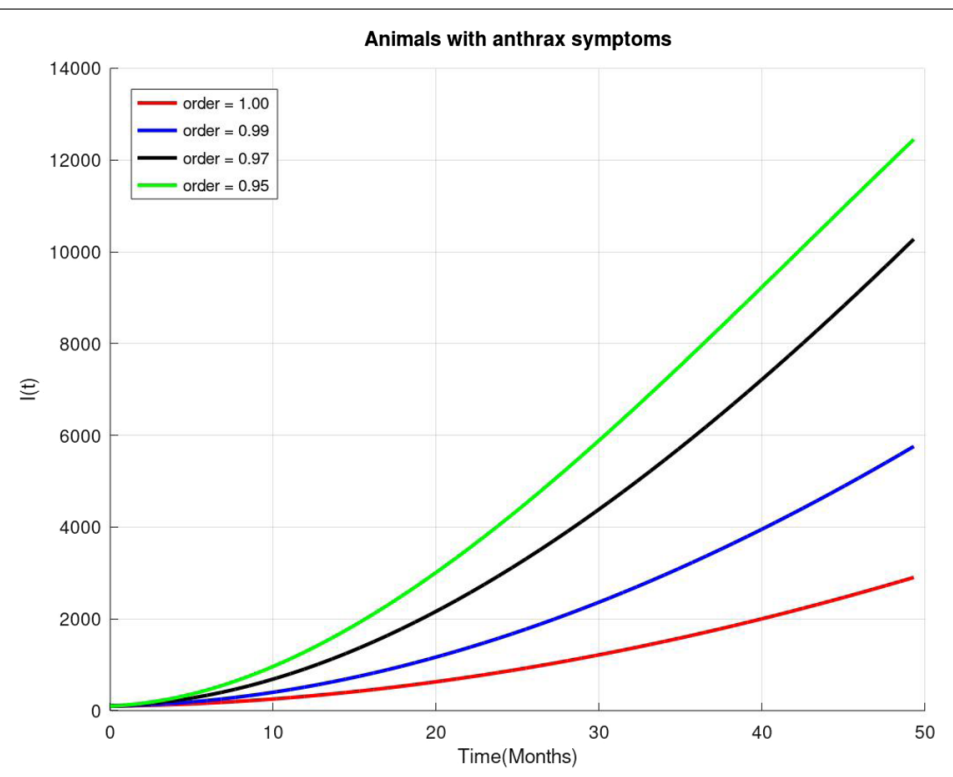

Figure 3 An illustration of the total number of infected animals $/(t)$ for different orders $\varrho=1, \varrho=0.99$, $\varrho=0.97$, and $\varrho=0.95$ during 50 months

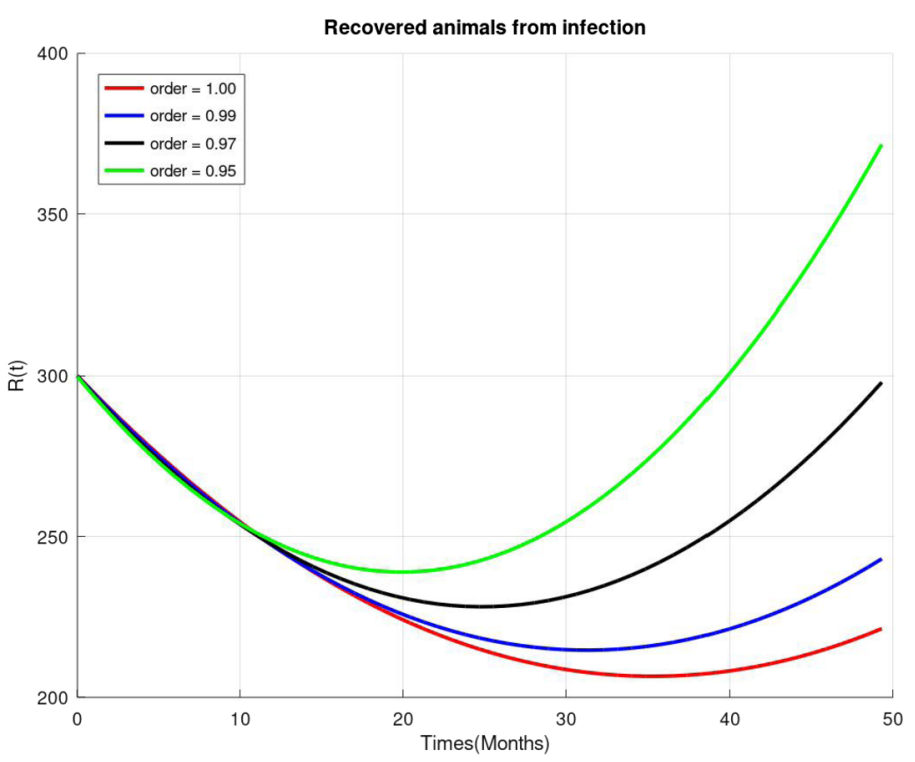

Figure 4 An illustration of the total number of recovered animals $R(t)$ for different orders $\varrho=1, \varrho=0.99$, $\varrho=0.97$, and $\varrho=0.95$ during 50 months

on the observations of this figure, we see that the total number of the susceptible animals increases by passing the time, this animal class is highly infected by anthrax disease, and the transmission rate of this disease remains high. In Figs. 2, 3, 4, 5, we plot the three-term approximate solutions of the fractional anthrax disease model by applying the homotopy analysis transform method (HATM) with auxiliary parameter $h=-1$ and auxiliary function $H(t)=1$ for different values of order $\varrho$. In each diagram, the solid red line illustrates the solution functions for integer order $\varrho=1$. These four Figs. 2, 3, 4, 5 indicate that by 


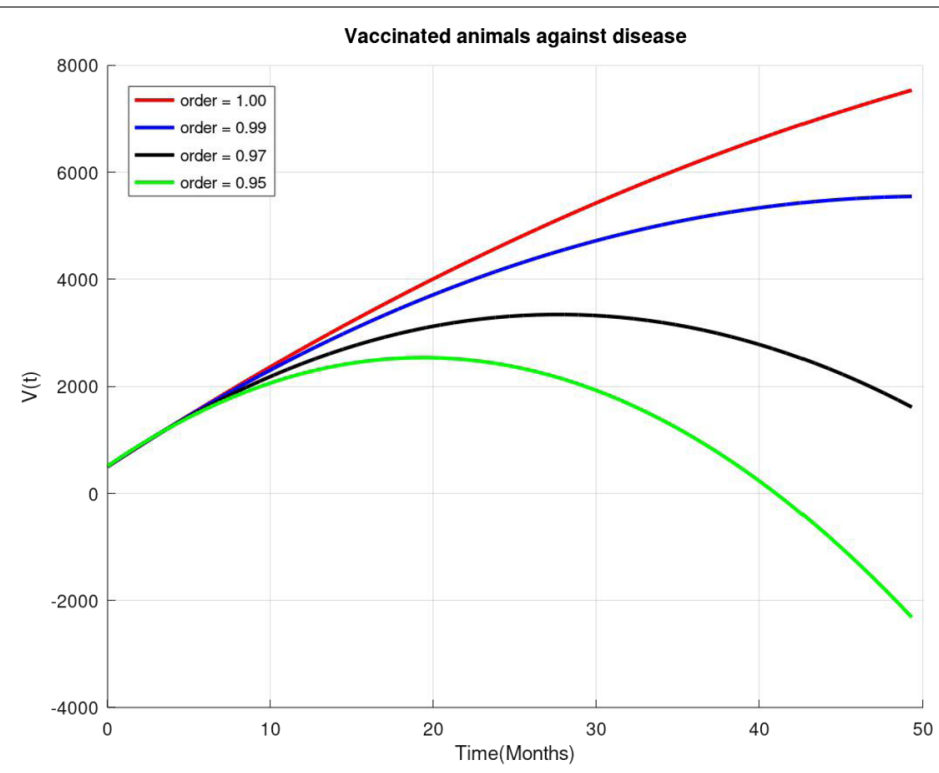

Figure 5 An illustration of the total number of vaccinated animals $V(t)$ for different orders $\varrho=1, \varrho=0.99$, $\varrho=0.97$, and $\varrho=0.95$ during 50 months

Table 1 Values of $S(t)$ for four different orders of ${ }^{\mathcal{C F}} \mathcal{D}_{0}^{\varrho}$

\begin{tabular}{llllllllll}
\hline$\varrho$ & $t=0$ & $t=3$ & $t=6$ & $t=9$ & $t=12$ & $t=15$ & $t=18$ & $t=21$ & $t=24$ \\
\hline 1 & 2000 & 2565.3 & 3148.6 & 3750 & 4369.8 & 5008.5 & 5666.3 & 6343.5 & 7040.4 \\
0.99 & 2001.6 & 2489.2 & 3010.9 & 3567.7 & 4160.6 & 4790.6 & 5458.7 & 6165.9 & 6913 \\
0.97 & 2003.2 & 2347.5 & 2759.6 & 3243.5 & 3803 & 4441.9 & 5164 & 5973.2 & 6873.2 \\
0.95 & 2002.9 & 2218.6 & 2539.1 & 2974.2 & 3533.5 & 4226.8 & 5063.8 & 6054.3 & 7208.1 \\
\hline
\end{tabular}

Table 2 Values of $/(t)$ for four different orders of ${ }^{\mathcal{F}} \mathcal{D}_{0}^{\varrho}$

\begin{tabular}{lllllccccc}
\hline$\varrho$ & $t=0$ & $t=3$ & $t=6$ & $t=9$ & $t=12$ & $t=15$ & $t=18$ & $t=21$ & $t=24$ \\
\hline 1 & 100 & 122.15 & 165.01 & 228.27 & 311.63 & 414.77 & 537.4 & 679.2 & 839.88 \\
0.99 & 100.06 & 139.14 & 222.51 & 349.19 & 518.19 & 728.54 & 979.23 & 1269.3 & 1597.8 \\
0.97 & 100.29 & 174.81 & 338.35 & 587.07 & 917.15 & 1324.8 & 1806 & 2357.2 & 2974.4 \\
0.95 & 100.73 & 213.06 & 455.07 & 817.03 & 1289.2 & 1861.8 & 2525.1 & 3269.4 & 4084.9 \\
\hline
\end{tabular}

Table 3 Values of $R(t)$ for four different orders of ${ }^{\mathcal{F}} \mathcal{D}_{0}^{\varrho}$

\begin{tabular}{llllllllll}
\hline$\varrho$ & $t=0$ & $t=3$ & $t=6$ & $t=9$ & $t=12$ & $t=15$ & $t=18$ & $t=21$ & $t=24$ \\
\hline 1 & 300 & 284.78 & 270.91 & 258.39 & 247.22 & 237.41 & 228.95 & 221.84 & 216.09 \\
0.99 & 299.94 & 284.36 & 270.35 & 257.91 & 247.04 & 237.74 & 230.02 & 223.86 & 219.27 \\
0.97 & 299.82 & 283.54 & 269.36 & 257.27 & 247.28 & 239.38 & 233.58 & 229.87 & 228.26 \\
0.95 & 299.68 & 282.74 & 268.57 & 257.16 & 248.51 & 242.62 & 239.5 & 239.13 & 241.53 \\
\hline
\end{tabular}

letting $\varrho \rightarrow 1$ the approximate solutions approach the classic integer solution with $\varrho=1$. More precisely, we provide Tables 1, 2, 3 and 4 for solutions $S(t), I(t), R(t)$, and $V(t)$, which represent a comparison between the obtained values for the fractional order $\mathcal{C F}$-model (8) with $\varrho=0.99, \varrho=0.97$, and $\varrho=0.95$ and the integer-order model (5) with $\varrho=1$. Based on this data, we find that the impact of the vaccination rate to control the spread of the an- 
Table 4 Values of $V(t)$ for four different orders of $\mathcal{C F}^{\mathcal{C}} \mathcal{D}_{0}^{\varrho}$

\begin{tabular}{llllllllll}
\hline$\varrho$ & $t=0$ & $t=3$ & $t=6$ & $t=9$ & $t=12$ & $t=15$ & $t=18$ & $t=21$ & $t=24$ \\
\hline 1 & 500 & 1083.9 & 1647.6 & 2191.2 & 2714.6 & 3217.8 & 3700.8 & 4163.6 & 4606.3 \\
0.99 & 502.03 & 1085.4 & 1633 & 2144.8 & 2620.9 & 3061.1 & 3465.6 & 3834.3 & 4167.3 \\
0.97 & 506.35 & 1088.3 & 1603.5 & 2052 & 2433.9 & 2749.1 & 2997.6 & 3179.5 & 3294.7 \\
0.95 & 511.06 & 1090.8 & 1573.1 & 1958 & 2245.4 & 2435.5 & 2528.2 & 2523.4 & 2421.3 \\
\hline
\end{tabular}

thrax disease between animals is vital, and hence this implies that the vaccination policies should be considered seriously to overcome this animal infection.

\section{Conclusions}

In this research work, we provide a fractional-order modeling of the anthrax disease between animals based on the Caputo-Fabrizio derivative. In the first step, we derive an existence criterion of solutions for proposed fractional $\mathcal{C F}$-system of the anthrax disease model by utilizing the Picard-Lindelof technique. Then by obtaining the basic reproduction number $\mathcal{R}_{0}$ of the fractional $\mathcal{C} \mathcal{F}$-system we compute two disease-free and endemic equilibrium points and check the asymptotic stability. Moreover, by applying an iterative approach based on the Sumudu transform, we investigate the stability of the fractional $\mathcal{C F}$-system. The approximate series solutions of this system are obtained by means of the homotopy analysis transform method, in which we invoke the linear Laplace transform. Finally, after the convergence analysis of numerical method HATM, we present a numerical simulation of the $\mathcal{C F}$-fractional anthrax disease model and review the dynamical behavior of the solutions of this $\mathcal{C F}$-system during a time interval.

Acknowledgements

The first and second authors were supported by University of Kasdi Merbah. The third and fourth authors were supported by Azarbaijan Shahid Madani University. The authors express their gratitude to anonymous referees for their helpful suggestions, which improved the final version of this paper.

Funding

Not applicable.

Availability of data and materials

Data sharing not applicable to this paper as no datasets were generated or analyzed during the current study.

Ethics approval and consent to participate

Not applicable.

Competing interests

The authors declare that they have no competing interests.

Consent for publication

Not applicable.

Authors' contributions

The authors declare that the study was realized in collaboration with equal responsibility. All authors read and approved the final manuscript.

\section{Author details}

${ }^{1}$ Institute of Research and Development, Duy Tan University, Da Nang 550000, Vietnam. ${ }^{2}$ Faculty of Natural Sciences, Duy Tan University, Da Nang 550000, Vietnam. ${ }^{3}$ Department of Medical Research, China Medical University Hospital, China Medical University, Taichung, Taiwan. ${ }^{4}$ Department of Mathematics, Azarbaijan Shahid Madani University, Tabriz, Iran.

${ }^{5}$ Department of Mathematics, Miandoab Branch, Islamic Azad University, Miandoab, Iran.

\section{Publisher's Note}

Springer Nature remains neutral with regard to jurisdictional claims in published maps and institutional affiliations. 


\section{References}

1. Baleanu, D., Jajarmi, A., Mohammadi, H., Rezapour, Sh.: A new study on the mathematical modelling of human liver with Caputo-Fabrizio fractional derivative. Chaos Solitons Fractals 134, Article ID 109705 (2020)

2. Baleanu, D., Mohammadi, H., Rezapour, Sh.: Analysis of the model of HIV-1 infection of CD4+ T-cell with a new approach of fractional derivative. Adv. Differ. Equ. 2020, Article ID 71 (2020)

3. Aydogan, S.M., Baleanu, D., Mousalou, A., Rezapour, Sh.: On high order fractional integro-differential equations including the Caputo-Fabrizio derivative. Bound. Value Probl. 2018, Article ID 90 (2018)

4. Baleanu, D., Mousalou, A., Rezapour, Sh.: On the existence of solutions for some infinite coefficient-symmetric Caputo-Fabrizio fractional integro-differential equations. Bound. Value Probl. 2017(1), Article ID 145 (2017). https://doi.org/10.1186/s13661-017-0867-9

5. Dokuyucu, M.A., Celik, E., Bulut, H., Baskonus, H.M.: Cancer treatment model with the Caputo-Fabrizio fractional derivative. Eur. Phys. J. Plus 133, Article ID 92 (2018)

6. Khan, S.A., Shah, G.Z.K., Jarad, F.: Existence theory and numerical solutions to smoking model under Caputo-Fabrizio fractional derivative. Chaos, Interdiscip. J. Nonlinear Sci. 29, Article ID 013128 (2019). https://doi.org/10.1063/1.5079644

7. Baleanu, D., Aydogan, S.M., Mohammadi, H., Rezapour, Sh.: On modelling of epidemic childhood diseases with the Caputo-Fabrizio derivative by using the Laplace Adomian decomposition method. Alex. Eng. J. (2020). https://doi.org/10.1016/j.aej.2020.05.007

8. Tuan, N.H., Mohammadi, H., Rezapour, Sh.: A mathematical model for COVID-19 transmission by using the Caputo fractional derivative. Chaos Solitons Fractals 140, Article ID 110107 (2020). https://doi.org/10.1016/j.chaos.2020.110107

9. Alzabut, J., Nieto, J.J., Stamov, G.Tr.: Existence and exponential stability of positive almost periodic solutions for a model of hematopoiesis. Bound. Value Probl. 2009, Article ID 127510 (2009). https://doi.org/10.1155/2009/127510

10. Matar, M.M., Amra, I.A., Alzabut, J.: Existence of solutions for tripled system of fractional differential equations involving cyclic permutation boundary conditions. Bound. Value Probl. 2020, Article ID 140 (2020), https://doi.org/10.1186/s13661-020-01437-x

11. Seemab, A., Ur Rahman, M., Alzabut, J., Hamdi, A.: On the existence of positive solutions for generalized fractional boundary value problems. Bound. Value Probl. 2019, Article ID 186 (2019). https://doi.org/10.1186/s13661-019-01300-8

12. Pratab, A., Alzabut, J., Dianavinnarasi, J., Cao, J., Rajchakit, G.: Finite-iime Mittag-Leffler stability of fractional-order quaternion-valued memristive neural networks with impulses. Neural Process. Lett. 51, 1485-1526 (2020). https://doi.org/10.1007/s11063-019-10154-1

13. Bozkurt, F., Yousef, A., Baleanu, D., Alzabut, J.: A mathematical model of the evolution and spread of pathogenic coronaviruses from natural host to human host. Chaos Solitons Fractals 138, Article ID 109931 (2020). https://doi.org/10.1016/j.chaos.2020.109931

14. Baleanu, D., Etemad, S., Rezapour, Sh.: On a fractional hybrid integro-differential equation with mixed hybrid integral boundary value conditions by using three operators. Alex. Eng. J. (2020). https://doi.org/10.1016/j.aej.2020.04.053

15. Baleanu, D., Rezapour, Sh., Mohammadi, H.: Some existence results on nonlinear fractional differential equations. Philos. Trans. R. Soc. A 2013, Article ID 20120144 (2013). https://doi.org/10.1098/rsta.2012.0144

16. Agarwal, R.P., Baleanu, D., Hedayati, V., Rezapour, Sh.: Two fractional derivative inclusion problems via integral boundary conditions. Appl. Math. Comput. 257, 205-212 (2015). https://doi.org/10.1016/j.amc.2014.10.082

17. Alsaedi, A., Baleanu, D., Etemad, S., Rezapour, Sh.: On coupled systems of time-fractional differential problems by using a new fractional derivative. J. Funct. Spaces 2016, Article ID 4626940 (2016). https://doi.org/10.1155/2016/4626940

18. Baleanu, D., Hedayati, V., Rezapour, Sh.: On two fractional differential inclusions. SpringerPlus 5, Article ID 882 (2016). https://doi.org/10.1186/s40064-016-2564-z

19. Samei, M.E., Yang, W.: Existence of solutions for k-dimensional system of multi-term fractional q-integro-differential equations under anti-periodic boundary conditions via quantum calculus. Math. Methods Appl. Sci. 43(7), 4360-4382 (2020). https://doi.org/10.1002/mma.6198

20. Ahmadian, A., Rezapour, Sh., Salahshour, S., Samei, M.E.: Solutions of sum-type singular fractional q-integro-differential equation with m-point boundary value problem using quantum calculus. Math. Methods Appl. Sci. (2020). https://doi.org/10.1002/mma.6591

21. Etemad, S., Rezapour, Sh., Samei, M.E.: On a fractional Caputo-Hadamard inclusion problem with sum boundary value conditions by using approximate endpoint property. Math. Methods Appl. Sci. (2020). https://doi.org/10.1002/mma.6644

22. Rezapour, Sh., Samei, M.E.: On the existence of solutions for a multi-singular pointwise defined fractional q-integro-differential equation. Bound. Value Probl. 2020, Article ID 38 (2020) https://doi.org/10.1186/s13661-020-01342-3

23. Samei, M.E., Rezapour, Sh.: On a system of fractional q-differential inclusions via sum of two multi-term functions on a time scale. Bound. Value Probl. 2020, Article ID 135 (2020). https://doi.org/10.1186/s13661-020-01433-1

24. Aydogan, S.M., Baleanu, D., Aguilar, J.F.G., Rezapour, Sh., Samei, M.E.: Approximate endpoint solutions for a class of fractional q-differential inclusions. Fractals 26(8), Article ID 2040029 (2020). https://doi.org/10.1142/S0218348X20400290

25. Baleanu, D., Rezapour, Sh., Saberpour, Z:: On fractional integro-differential inclusions via the extended fractional Caputo-Fabrizio derivation. Bound. Value Probl. 2019, Article ID 79 (2019)

26. Baleanu, D., Etemad, S., Rezapour, Sh.: A hybrid Caputo fractional modeling for thermostat with hybrid boundary value conditions. Bound. Value Probl. 2020, Article ID 64 (2020). https://doi.org/10.1186/s13661-020-01361-0

27. Castillo-Chavez, C., Feng, Z., Huang, W.: On the computation of $\mathcal{R}_{0}$ and its role on global stability. IMA Vol. Math. Appl. $125,229-250(2002)$

28. Wendy, C.T.: Soil ingestion, nutrition and seasonality of anthrax in herbivores of Etosha National Park. Ecosphere 4(1), $1-19(2013)$

29. Osman, S., Oluwole, D.M., Theuri, D.M.: Mathematical modelling of transmission dynamics of anthrax in human and animal population. Math. Theory Model. 8(6), 47-67 (2018) 
30. Anderson, R.M., May, R.M.: Population biology of infectious diseases: part 1. Nature 280, 361-367 (1979). https://doi.org/10.1038/280361a0

31. Gutting, B.W., Channel, S.R., Berger, A.E., Gearhart, J.M., Andrews, G.A., Sherwood, R.L., Nichols, T.L.: Mathematica modelling of transmission dynamics of anthrax in human and animal population. Microbe 3(2), $78-85$ (2008)

32. Mushayabasa, S.: Global stability of an anthrax model with environmental decontamination and time delay. Discrete Dyn. Nat. Soc. 2015, Article ID 573146 (2015). https://doi.org/10.1155/2015/573146

33. Sinkie, Z.M., Murthy, N.S.: Modeling and simulation study of anthrax attack on environment. J. Multidiscip. Eng. Sci. Technol. 3(4), 4574-4578 (2016)

34. Friedman, A., Yakubu, A.A.: Anthrax epizootic and migration: persistence or extinction. Math. Biosci. 241(1), 137-144 (2013). https://doi.org/10.1016/j.mbs.2012.10.004

35. Hahn, B.D., Furniss, P.R.: A deterministic model of anthrax epizootic threshold results. Ecol. Model. 20(2-3), 233-241 (1983)

36. Mushayabasa, S., Marijani, T., Masocha, M.: Dynamical analysis and control strategies in modeling anthrax. Comput. Appl. Math. 36(3), 1333-1348 (2017)

37. Kimathi, G., Wainaina, M.: Analysis of transmission dynamics of anthrax in animals: a modeling approach. J. Sci. Res. Rep. 23(1), Article ID JSRR.47644 (2019)

38. Khuri, S.A.: A Laplace decomposition algorithm applied to a class of nonlinear differential equations. J. Appl. Math. 1(4), 141-155 (2001)

39. Liao, S.J.: Beyond Perturbation: Introduction to Homotopy Analysis Method. Chapman \& Hall, New York (2003)

40. Rida, S.Z., Arafa, A.A.M., Gaber, Y.A.: Solution of the fractional epidemic model by L-ADM. J. Fract. Calc. Appl. 7(1), 189-195 (2016)

41. Podlubny, I.: Fractional Differential Equations. Academic Press, New York (1999)

42. Samko, G., Kilbas, A., Marichev, O.: Fractional Integrals and Derivatives: Theory and Applications. Gordon \& Breach, Yverdon (1993)

43. Caputo, M., Fabrizio, M.: A new definition of fractional derivative without singular kernel. Prog. Fract. Differ. Appl. 1(2), 73-85 (2015)

44. Losada, J., Nieto, J.J.: Properties of the new fractional derivative without singular kernel. Prog. Fract. Differ. Appl. 1(2), 87-92 (2015)

45. Belgacem, F.B.M., Karaballi, A.A., Kalla, S.L.: Analytical investigations of the Sumudu transform and applications to integral production equations. Math. Probl. Eng. 23(3), 103-118 (2003)

46. Bodkhe, D.S., Panchal, S.K.: On Sumudu transform of fractional derivatives and its applications to fractional differential equations. Asian J. Math. Comput. Res. 11(1), 69-77 (2016)

47. Shah, K., Junaid, M., Ali, N.: Extraction of Laplace, Sumudu, Fourier and Mellin transform from the natural transform. J. Appl. Environ. Biol. Sci. 5(9), 108-115 (2015)

48. Watugala, G.K.: Sumudu transform: a new integral transform to solve differential equations and control engineering problems. Int. J. Math. Educ. Sci. Technol. 24(1), 35-43 (1993)

49. Wang, J., Zhou, Y., Medved, M.: Picard and weakly Picard operators technique for nonlinear diferential equations in Banach spaces. J. Math. Anal. Appl. 389, 261-274 (2012)

50. Li, H., Cheng, J., Li, H.B., Zhong, S.M.: Stability analysis of a fractional-order linear system described by the Caputo-Fabrizio derivative. Mathematics 7(2), Article ID 200 (2019)

51. Van den Driessche, P., Watmough, J.: Reproduction numbers and subthreshold endemic equilibria for compartmenta models of disease transmission. Math. Biosci. 180, 29-48 (2002)

52. Arqub, O.A., El-Ajou, A.: Solution of the fractional epidemic model by homotopy analysis method. J. King Saud Univ., Sci. 25(1), 73-81 (2013)

53. Rafei, M., Ganji, D.D., Daniali, H.: Solution of the epidemic model by homotopy perturbation method. Appl. Math. Comput. 187(2), 1056-1062 (2007)

\section{Submit your manuscript to a SpringerOpen ${ }^{\circ}$ journal and benefit from:}

- Convenient online submission

- Rigorous peer review

- Open access: articles freely available online

- High visibility within the field

- Retaining the copyright to your article

Submit your next manuscript at $>$ springeropen.com 